\title{
A large and functionally diverse family of Fad2 genes in safflower (Carthamus tinctorius L.)
}

\author{
Shijiang Cao ${ }^{1,2}$, Xue-Rong Zhou', Craig C Wood ${ }^{1}$, Allan G Green', Surinder P Singh ${ }^{1}$, Lixia Liu²* and Qing Liu*
}

\begin{abstract}
Background: The application and nutritional value of vegetable oil is highly dependent on its fatty acid composition, especially the relative proportion of its two major fatty acids, i.e oleic acid and linoleic acid. Microsomal oleoyl phosphatidylcholine desaturase encoded by FAD2 gene is known to introduce a double bond at the $\Delta 12$ position of an oleic acid on phosphatidylcholine and convert it to linoleic acid. The known plant FAD2 enzymes are encoded by small gene families consisting of 1-4 members. In addition to the classic oleate $\Delta 12$-desaturation activity, functional variants of FAD2 that are capable of undertaking additional or alternative acyl modifications have also been reported in a limited number of plant species. In this study, our objective was to identify FAD2 genes from safflower and analyse their differential expression profile and potentially diversified functionality.

Results: We report here the characterization and functional expression of an exceptionally large FAD2 gene family from safflower, and the temporal and spatial expression profiles of these genes as revealed through Real-Time quantitative PCR. The diversified functionalities of some of the safflower FAD2 gene family members were demonstrated by ectopic expression in yeast and transient expression in Nicotiana benthamiana leaves. CtFAD2-1 and CTFAD2-10 were demonstrated to be oleate desaturases specifically expressed in developing seeds and flower head, respectively, while CtFAD2-2 appears to have relatively low oleate desaturation activity throughout the plant. CtFAD2-5 and CtFAD2-8 are specifically expressed in root tissues, while CtFAD2-3, 4, 6, 7 are mostly expressed in the cotyledons and hypocotyls in young safflower seedlings. CtFAD2-9 was found to encode a novel desaturase operating on C16:1 substrate. CtFAD2-11 is a tri-functional enzyme able to introduce a carbon double bond in either cis or trans configuration, or a carbon triple (acetylenic) bond at the $\triangle 12$ position.

Conclusions: In this study, we isolated an unusually large FAD2 gene family with 11 members from safflower. The seed expressed FAD2 oleate $\Delta 12$ desaturase genes identified in this study will provide candidate targets to manipulate the oleic acid level in safflower seed oil. Further, the divergent FAD2 enzymes with novel functionality could be used to produce rare fatty acids, such as crepenynic acid, in genetically engineered crop plants that are precursors for economically important phytoalexins and oleochemical products.
\end{abstract}

\section{Background}

Safflower (Carthamus tinctorius L.) is an ancient oilseed crop that is currently grown for its high quality edible oil used in cooking, salad dressings and margarines, and to a lesser degree as a bird seed. The characteristics of oils are highly dependent on their fatty acid composition. Oleic acid $\left(\mathrm{C} 18: 1^{\Delta 9}\right)$ and linoleic acid $\left(\mathrm{C} 18: 2^{\Delta 9,12}\right)$ are the two major fatty acids found in safflower seed oil,

\footnotetext{
* Correspondence: liulx062@nenu.edu.cn; qing.liu@csiro.au

${ }^{2}$ School of Life Sciences, Northeast Normal University, Changchun, China ${ }^{1}$ Commonwealth Scientific and Industrial Research Organization Plant Industry, PO Box 1600, Canberra ACT 2601, Australia

Full list of author information is available at the end of the article
}

together accounting for about $90 \%$ of the total fatty acids. Conventional safflower oil is characterised by its relatively high level of linoleic acid content about $70 \%$ compared to most other oilseed crops [1]. In the past six decades, breeders have exploited safflower's natural genetic diversity to modify the oleate/linoleate ratio for particular end use purposes. Numerous breeding lines with high levels of either oleic acid (75-84\%) or linoleic acid (71-89\%) have been selected. The relative proportions of these two major fatty acids determine relevant technological and nutritional properties of edible oils [2]. Nutritionally, both oleic acid and linoleic acid can lower total serum cholesterols, however, oleic acid has

\section{Biomed Central}


higher oxidative stability compared to linoleic acid as it contains one less double bond. Therefore raising oleic acid content at the expense of linoleic acid has been set as an important research objective for the improvement of many oilseed crops, including safflower, to provide highly stable cooking oils without the need for hydrogenation, a process that can result in the formation of nutritionally undesirable trans fatty acid [3,4]. Beyond food applications, high oleic oils also have significant existing and potential industrial uses, such as in improved biodiesel, lubricants, and hydraulic oils because of the high oxidative stability needed in these products. Purified oleic acid is also a valuable industrial chemical feedstock, and can be cleaved to form derivatives such as azelaic acid that can be used in the formulation of a range of industrial products and polymers [5-7].

The distinct fatty acid compositions found in seed storage oil and membrane lipids are the result of an intricate metabolic network that regulates fatty acid biosynthesis and flux through both prokaryotic and eukaryotic pathways $[8,9]$. It is understood that the chloroplast $\Delta 12$ desaturase (FAD6) is necessary for desaturating 16:1 and 18:1 fatty acids to $16: 2$ and $18: 2$ on all $16: 1$ - or $18: 1$-containing chloroplast membrane lipids including phosphatidyl glycerol (PG), monogalactosyldiacylglycerol (MGDG), digalactosyldiaclyglycerol (DGDG), and sulfoguinovosyldiacylglycerol (SQDG) [9]. The enzyme primarily responsible for the synthesis of linoleic acid from oleic acid in seed storage lipids is the microsomal oleoyl phosphatidylcholine desaturase (FAD2) that introduces a double bond at the $\Delta 12$ position of oleic acid on phosphatidylcholine (PC), forming linoleic acid on the endoplasmic reticulum (ER) [10-12]. Variants of the FAD2 enzyme are also known to have diversified functionalities in fatty acid modification, catalysing hydroxylation [13,14], epoxidation [15], and the formation of acetylenic bonds $[15,16]$ and conjugated double bonds [17-20]. Some functionally divergent FAD2 enzymes are multi-functional, such as the bifunctional hydroxylase/desaturase from Lesquerella fendleri [21], and tri-functional acetylenase from Crepis alpina, which can also catalyse the formation of both trans and cis double bonds at the $\Delta 12$ position of oleic acid [22].

Membrane integrity and function, determined by structure and fluidity, are largely affected by lipid composition and the degree of fatty acid desaturation in plants [23]. Because the FAD2 enzyme is the key step in the accumulation of polyunsaturated fatty acids it plays an essential role in the biophysical characteristics of cell membranes and is often induced in response to various environmental stimuli such as extreme temperatures [8], high salt [24], and pathogen attack [25]. The expression of FAD2 leading to the production of polyunsaturated fatty acids is also important to the specific signal transduction pathways, such as jasmonic acid pathway that is known to be a critical factor in plant defence system and male fertility [26-28].

Since the cloning of first plant FAD2 gene in Arabidopsis thaliana [11], its orthologous DNA sequences have been isolated and characterised from many different plant species, including soybean [29], rape [30], cotton [31-34], peanut [35,36] and flax [37]. Only a single FAD2 gene exists in Arabidopsis, but in most other plant species FAD2 is encoded by small gene families. For example, FAD2 is encoded by three distinct family members in soybean $[29,38]$ and by four members in cotton [31-34]. Here we report the discovery, isolation and characterisation of an unprecedentedly large FAD2 gene family from safflower. Phylogenetic analysis of eleven full length cDNAs and their distinct genomic structural features indicated that they are non-allelic and have likely evolved through gene duplication at several hierarchical levels. Their distinct expression patterns were revealed by real time quantitative PCR (RT-qPCR) of different safflower tissues. Functional divergence of the FAD2 family members was explored by heterologous expression in yeast and transient expression in Nicotiana benthamiana.

\section{Results}

Cloning and sequencing analysis of multiple members of safflower CtFAD2 gene family

Two different full length cDNAs, designated as CtFAD2-1 and CtFAD2-2, were isolated from the lambda cDNA library derived from safflower developing embryos, using Arabidopsis FAD2 DNA sequence as a probe. The Arabidopsis FAD2 DNA sequence was also used to "blast" search the Expressed Sequence Tags (ESTs) database generated by the Compositae Genome Project (CGP, http://compgenomics.ucdavis.edu/compositae_index.php). From the total 41,317 safflower ESTs at the time we searched, at least eleven distinct FAD2 cDNA sequence contigs were identified and designated. In addition to CtFAD2-1 and CtFAD2-2 that had already been isolated from the developing embryo cDNA library, a further 9 partial cDNAs were designated as CtFAD2-3 through to $C t F A D 2-11$, respectively. Gene specific primers were designed from each of the partial cDNA sequences, and the full length cDNA were obtained by conducting $3^{\prime}$ and $5^{\prime}$ rapid amplification of cDNA ends (RACE) reaction on RNAs derived from various safflower plant tissues, including leaf, root, hypocotyl and flower head. The amplified fragment was subcloned into the pGEM-Teasy vector and sequenced from both directions. Sequence comparisons of the $3^{\prime}$ and $5^{\prime}$ ends with the corresponding ESTs showed overlapping regions that matched with each other. Subsequently full length cDNAs encoding the 9 distinct partial FAD2 ESTs were obtained by assembling the RACE product with corresponding ESTs. Each cDNA 
contains an uninterrupted coding region that shares extensive sequence homology with each other, and unique $5^{\prime}$ and $3^{\prime}$ untranslated region (UTR) sequences. The putative polypeptides were varied between 372-388 amino acids. The sequence similarity among these 11 CtFAD2 coding regions at both nucleotide and amino acid levels is listed in Table 1. It was also revealed that the putative polypeptides of the 11 CtFAD2 genes share about 50\%-65\% sequence identity to orthologous FAD2 enzymes in other plants.

To elucidate phylogenetic relationship of safflower FAD2 genes, the 11 deduced polypeptide sequences were aligned with orthologous FAD2 sequences and a neighbour-joining tree was constructed using Vector NTI. As shown in Figure 1, CtFAD2-1 and CtFAD2-10 are aligned next to each other and to other seed expressed FAD2s, such as sunflower HaFAD2-1 and cotton GhFAD2-1. CtFAD2-2 is aligned together with other constitutively expressed genes, such as sunflower HaFAD2-2 and HaFAD2-3. CtFAD2-3, 4, 5, 6 and 7 form a new branch, most likely to have recently become divergent. Interestingly it is embedded deep in a clade with functionally divergent FAD2 fatty acid modifying enzymes, such as fatty acid acetylenases and epoxygenases. CtFAD2-11 is also aligned together with fatty acid acetylenases from several plant species, including the sunflower vFAD2 that was induced by fungal elicitors [39].

The alignment of the putative polypeptide sequences of CtFAD2s together with the selected plant orthologs is given in (Additional file 1: Figure S1). Similar to other plant FAD2, the putative polypeptides of CtFAD2s contain an aromatic amino acid-rich motif, at the C-terminus, which is both necessary and sufficient for maintaining localization in the ER [40]. Consistent with other plant membrane bound fatty acid desaturase enzymes, the putative polypeptides deduced from all eleven safflower CtFAD2 cDNAs contain three highly conserved histidine-rich motifs that have been implicated in the formation of the diiron-oxygen complex used in biochemical catalysis [41]. The first histidine motif is HECGHH in majority of CtFAD2 putative polypeptide sequences, except CtFAD2-5 and -6 that have HDCGHH, HDLGHH, respectively. The last amino acid His $(\mathrm{H})$ has been converted to a $\mathrm{Gln}(\mathrm{Q})$ in CtFAD-8 (HECGHQ). We have compared this motif in 55 plant FAD2 enzymes and the $\mathrm{H}$ to $\mathrm{Q}$ substitution is also present in a diverged FAD2 homologue from Lesquerella lindheimeri with predominantly fatty acid hydroxylase activity [42]. The second histidine motif is highly conserved as HRRHH in several safflower FAD2s, including CtFAD2-1, 2, 8, 9 and 10. It is noteworthy that at +3 of the motif an Asn (N) substitution was found in CtFAD2-11, consistent with a number of functionally divergent FAD2s, including Crepis alpina CREP1 (ABC00769), C. palaestina Cpal2 (CAA76156) and sunflower vFAD2 (AY166773.1), Calendula officinalis FAC2 (AF343064.1), Rudbeckia hirta acetylenase (AY166776.1). Either a Ser (S) or Thr (T) substitution spotted at this position in CtFAD2-3, 4, 5, 6 and 7.

In CtFAD2-1, 2, 9, 10 the amino acid immediately preceding the first histidine box is an Ala (A), consistent with other plant FAD2 $\Delta 12$ oleate desaturase enzymes. Ala substitution by Val (V) occurred in CtFAD2-5, while other six CtFAD2 enzymes have Gly (G) in this position. It was proposed by Cahoon et al. [39] that Gly substitution of Ala at this position has been consistently found in functionally divergent FAD2 enzymes, except $\Delta 12$ hydroxylase. It is noted that only CtFAD2-11 has a DVTH motif, in the -5 to -2 positions of the third Histidine box, which fits with the $(\mathrm{D} / \mathrm{N}) \mathrm{VX}(\mathrm{H} / \mathrm{N})$ motif

Table 1 Sequence identity of the coding region DNA and deduced amino acids in safflower CtFAD2 genes

\begin{tabular}{|c|c|c|c|c|c|c|c|c|c|c|c|c|}
\hline & & \multicolumn{11}{|c|}{ Deduced amino acids } \\
\hline & & $\begin{array}{c}C t F A D 2- \\
1\end{array}$ & $\begin{array}{c}\text { CtFAD2- } \\
2\end{array}$ & $\begin{array}{c}\text { CtFAD2- } \\
3\end{array}$ & $\begin{array}{c}\text { CtFAD2- } \\
4\end{array}$ & $\begin{array}{c}C t F A D 2- \\
5\end{array}$ & $\begin{array}{c}C t F A D 2- \\
6\end{array}$ & $\begin{array}{c}\text { CtFAD2- } \\
7\end{array}$ & $\begin{array}{c}\text { CtFAD2- } \\
8\end{array}$ & $\begin{array}{c}\text { CtFAD2- } \\
9\end{array}$ & $\begin{array}{c}\text { CtFAD2- } \\
10\end{array}$ & $\begin{array}{c}C t F A D 2- \\
11\end{array}$ \\
\hline \multirow{11}{*}{$\begin{array}{l}\text { Nucleotide } \\
\text { acid }\end{array}$} & CtFAD2-1 & - & $70.3 \%$ & $53.2 \%$ & $52.5 \%$ & $53.5 \%$ & $50.9 \%$ & $54.1 \%$ & $59.7 \%$ & $59.5 \%$ & $80.1 \%$ & $56.4 \%$ \\
\hline & CtFAD2-2 & $70.0 \%$ & - & $54.5 \%$ & $55.0 \%$ & $54.2 \%$ & $51.7 \%$ & $57.8 \%$ & $60.6 \%$ & $62.5 \%$ & $69.5 \%$ & $58.6 \%$ \\
\hline & CtFAD2-3 & $62.0 \%$ & $62.0 \%$ & - & $97.1 \%$ & $62.0 \%$ & $61.8 \%$ & $63.1 \%$ & $52.7 \%$ & $50.9 \%$ & $51.2 \%$ & $56.8 \%$ \\
\hline & CtFAD2-4 & $62.7 \%$ & $63.3 \%$ & $95.1 \%$ & - & $61.4 \%$ & $61.4 \%$ & $63.3 \%$ & $53.1 \%$ & $50.9 \%$ & $50.9 \%$ & $56.9 \%$ \\
\hline & CtFAD2-5 & $61.9 \%$ & $60.3 \%$ & $69.7 \%$ & $70.6 \%$ & - & $63.2 \%$ & $62.0 \%$ & $51.4 \%$ & $51.3 \%$ & $51.7 \%$ & $53.9 \%$ \\
\hline & CtFAD2-6 & $60.6 \%$ & $59.9 \%$ & $68.8 \%$ & $69.6 \%$ & $72.0 \%$ & - & $63.1 \%$ & $49.3 \%$ & $50.8 \%$ & $50.1 \%$ & $56.2 \%$ \\
\hline & CtFAD2-7 & $62.2 \%$ & $65.8 \%$ & $69.4 \%$ & $69.3 \%$ & $66.6 \%$ & $68.2 \%$ & - & $51.7 \%$ & $49.2 \%$ & $51.4 \%$ & $60.7 \%$ \\
\hline & CtFAD2-8 & $65.2 \%$ & $66.2 \%$ & $63.1 \%$ & $62.8 \%$ & $60.8 \%$ & $61.7 \%$ & $61.2 \%$ & - & $58.8 \%$ & $58.1 \%$ & $56.40 \%$ \\
\hline & CtFAD2-9 & $64.9 \%$ & $66.2 \%$ & $59.5 \%$ & $59.5 \%$ & $58.3 \%$ & $59.2 \%$ & $59.5 \%$ & $63.5 \%$ & - & $59.3 \%$ & $55.9 \%$ \\
\hline & CtFAD2-10 & $78.9 \%$ & $72.0 \%$ & $60.7 \%$ & $62.0 \%$ & $59.8 \%$ & $61.0 \%$ & $60.7 \%$ & $64.3 \%$ & $64.1 \%$ & - & $57.2 \%$ \\
\hline & CtFAD2-11 & $60.0 \%$ & $62.9 \%$ & $64.1 \%$ & $64.4 \%$ & $62.4 \%$ & $64.1 \%$ & $62.7 \%$ & $63.9 \%$ & $60.9 \%$ & $61.7 \%$ & - \\
\hline
\end{tabular}




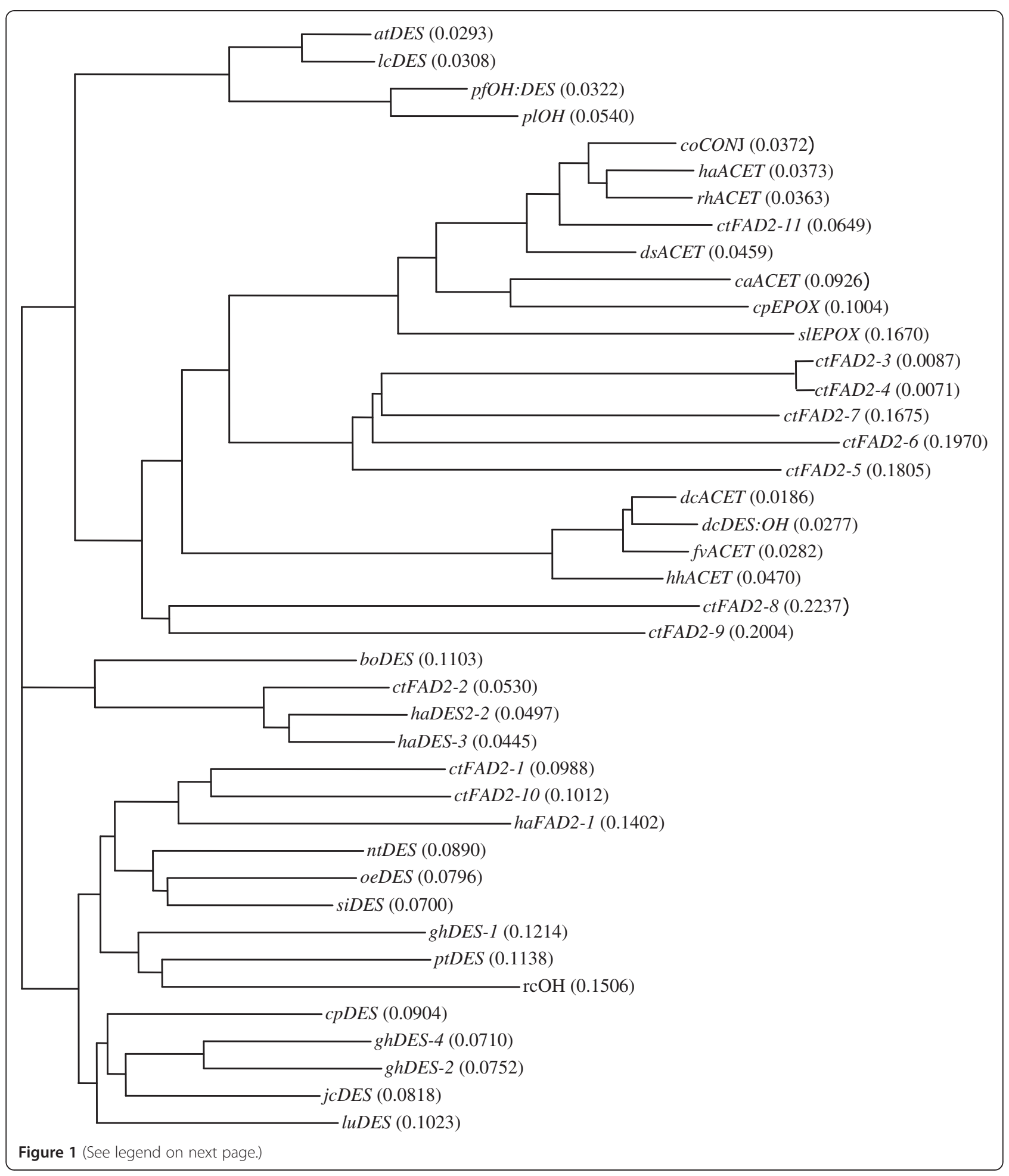


(See figure on previous page.)

Figure 1 Phylogenetic comparison of safflower CtFAD2 gene family and orthologous FAD2s from other plants. The phylogenetic tree was generated by Vector NTI (invitrogen). Included in the alignment were FAD2 desaturases (DES), hydroxylases (OH), epoxygenases (EPOX), acetylenases (ACET), and conjugases (CONJ). The GenBank accession numbers of the amino acid sequences represented in the phylogenetic tree are: atDES, AAM61113.1; ICDES, ACR15954.1; pfOH:DES, AAC32755.1; plOH, ABQ01458.1; coCONJ, AAK26632.1; haACET, ABC59684.1; rhACET, AAO38035.1; dsACET, AAO38036.1; CaACET, ABC00769.1; cPEPOX, CAA76156.1; sIEPOX, AAR23815.1; dCACET, AAO38033.1; dCDES:OH, AAK30206.1; fvACET,AAO38034.1; h hACET, AAO38031.1; boDES, AAC31698.1; haDES-2, AAL68982.1; haDES-3, AAL68983.1; haDES-1, AAL68981.1; ntDES, AAT72296.2; oeDES, AAW63040; siDES, AAF80560.1; ghDES-1, CAA65744.1; ptDES, XP_002297660.1; rcOH, AAC49010.1; cPDES, AAS19533.1; ghDES-4, AAQ16653.1; ghDES-2, CAA71199.1; jCDES, ADB93805.1; luDES, ACF49507.1. (at, Arabidopsis thaliana; lc, Lepidium campestre; pf, Physaria fendleri; pl, Physaria lindheimeri; co, Calendula officinalis; ha, Helianthus annuus; rh, Rudbeckia hirta; ds, Dimorphotheca sinuate; ca, Crepis alpine; cp, Crepis palaestina; sl, Stokesia laevis; dc, Daucus carota; fv, Foeniculum vulgare; hh, Hedera helix; Bo, Borago officinalis; nt, Nicotiana tabacum; oe, Olea europaea; si, Sesamum indicum; gh, Gossypium hirsutum; pt, Populus trichocarpa; rc, Ricinus communis; cp, Cucurbita pepo; jc, Jatropha curcas; lu, Linum usitatissimum).

proposed to occur in all plant acetylenases [43]. The five amino acids immediately behind the third histidine box of CtFAD2-1, 2, 10, are LFSTM as for other known plant FAD2 $\Delta 12$ oleate desaturases. In contrast, CtFAD2-9 has an LFSYI motif at this position with two amino acid substitutions at +4 and +5 position. In CtFAD2-3, 4 and 7 , the $S$ at the +3 position is substituted by Pro $(\mathrm{P})$, which is also exclusively present in other FAD2 fatty acid conjugases, including those from Calendula officinalis (FAC2, Genbank AAK26632) and Trichosanthes kirilowii (Genbank AAO37751).

It was shown that the Serine-185 of soybean seedspecific GmFAD2-1 sequences is phosphorylated during seed development [44], as a regulatory mechanism for its enzymatic activity. Among the 11 safflower FAD2 enzymes only CtFAD2-1, the seed-specific FAD2, has the Serine corresponding with that in soybean FAD2-1. It is tempting to predict that the same posttranslational regulatory mechanism of FAD2 expression through phosphorylation of target site including the Serine-185 may play an important role in modulating microsomal $\Delta 12$ oleate desaturation during safflower seed development and oil accumulation.

\section{Genomic structures and evolutionary studies of the CtFAD2 gene family in safflower}

In the context of elucidating the gene structure of FAD2 from safflower, an intron that is situated in the $5^{\prime}$ UTR of FAD2 genes was isolated. From each of the 11 safflower FAD2 cDNA sequences, the typical splice site (AG:GT) was predicted and PCR primers were designed for amplification of such an intron from genomic DNA. The predicted $5^{\prime}$ intron was obtained from 8 of the 11 CtFAD2 genes, including CtFAD2-1, -2, -3, -4, -5, -7, -10 and -11 . The major features of the primary DNA sequences of these CtFAD2 introns are summarised in Table 2. The intron was not amplified from CtFAD2-6, -8 and -9 , likely because the $5^{\prime}$ UTR in which the introns reside is incomplete in our clones. All of the eight intron sequences were located within the $5^{\prime}$ UTR, at positions that varied between 11 to $38 \mathrm{bp}$ upstream of the putative start codon, the first ATG. The intron length varied between 114 to 3,090 bp and was 1,144 bp for CtFAD2-1, similar in size to the introns identified in FAD2 genes from Arabidopsis (The Arabidopsis Information Resource, http://www.arabidopsis.org), cotton [45] and sesame (Sesamum indicum) [46]. The variations in the relative positions and the substantial differences in the sizes of the $5^{\prime}$-UTR introns are distinguishing structural differences among safflower FAD2 genes, which could be important in differential expression of the genes. The large intron present in the $5^{\prime}$-UTR of FAD2 genes could play an important role in expression regulation, as it has been reported to have positive effects on the expression of reporter genes in sesame [46]. The equivalent intron has also been shown to be an effective target for posttranscriptional gene silencing of FAD2 in soybean [47].

Table 2 The features of CtFAD2 gene introns

\begin{tabular}{|c|c|c|c|c|c|c|}
\hline & Position & Length & AT content & CG content & $5^{`} \mathrm{E} / \mathrm{l}$ boundary & $3^{`} \mathrm{I} / \mathrm{E}$ boundary \\
\hline CtFAD2-1 & -13 & 1144 & $64.5 \%$ & $35.5 \%$ & AG:GTGCAT & TTGCAG:GT \\
\hline CtFAD2-2 & -12 & 3090 & $65.8 \%$ & $34.2 \%$ & AG:GTGAGA & TTGCAG:GT \\
\hline CtFAD2-3 & -11 & 114 & $73.7 \%$ & $26.3 \%$ & AG:GTATGA & ATGCAG:GT \\
\hline CtFAD2-4 & -11 & 124 & $75.0 \%$ & $25.0 \%$ & AG:GTAAGT & GCGCAG:GT \\
\hline CtFAD2-5 & -33 & 96 & $69.8 \%$ & $30.2 \%$ & AG:GTACCT & TITCAG:GT \\
\hline CtFAD2-7 & -29 & 242 & $61.6 \%$ & $38.4 \%$ & AG:GTATAC & TTGCAG:GT \\
\hline CtFAD2-10 & -38 & 2247 & $68.9 \%$ & $31.1 \%$ & TG:GTTCGT & TTACAG:GT \\
\hline CtFAD2-11 & -22 & 334 & $62.5 \%$ & $37.5 \%$ & AG:CTCACA & GTCTT:GT \\
\hline
\end{tabular}


The putative splice sites, at both $5^{\prime}$ exon/intron and $3^{\prime}$ intron/exon, are conserved as AG:GT in most of the eight CtFAD2 genes, except the $5^{\prime}$ exon/intron (TG:GT) in CtFAD2-10, and both the $5^{\prime}$ exon/intron (AG:CT) and $3^{\prime}$ intron/exon (TT:GT) in CtFAD2-11. However, the intron sequences themselves are all highly divergent without any significant homology among them. These intron sequences are AT-rich with AT content between $61 \%$ and 75\%, consistent with other dicot intron sequences. Analyzing the intron sequences by the PLACE program (http://www. dna.affrc.go.jp/PLACE/) identified several putative cisregulatory elements. For instance, a few motifs, such as ABRE and SEF4, commonly present in seed-specific promoters were located in the seed-specific CtFAD2-1. Likewise, an AG-motif that is often present in the promoter of defence-related genes induced by various stresses, such as wounding or elicitor treatment, was located in the CtFAD2-3 gene that is specifically expressed in the hypocotyls and cotyledons of young safflower seedlings.

\section{Analysis safflower CtFAD2 gene family by Southern blot analysis}

The complexity of the FAD2 gene family in safflower was also examined by low stringency Southern blot analysis and confirmed that safflower FAD2 is encoded by a complex multigene family (Figure 2). The restriction fragment patterns derived from various restriction enzymes are consistent with there being more than 10 $F A D 2$ genes in safflower. The variation in the intensity of hybridization signals reflects the relative levels of sequence similarity with the probe DNA used.

\section{Expression profile of CtFAD2 genes in different tissues}

To determine tissue expression patterns of the various CtFAD2 genes, RT-PCR analysis was carried out with total RNA extracted from cotyledon, hypocotyl, root and leaf tissues derived from young seedlings, as well as flower tissues and developing embryos of safflower genotype SU. No product was detectable after 40 cycles of amplification in control reactions without reverse transcriptase, demonstrating the absence of genomic DNA in RNA samples.

As shown in Figure 3, CtFAD2-1 is specifically expressed in developing seeds, with little if any expression detected in the somatic tissues examined. CtFAD2-2 is expressed at low levels in seeds as well as in all other tissues examined. CtFAD2-4, -5, -6, -7, -8, -9 showed no expression in developing embryos. Low, yet detectable levels of CtFAD2-10 and -11 expression were observed in developing seeds, especially in the late development stage. CtFAD2-4, -6, -7, -9 and -11 showed high expression in young seedling tissues including cotyledons and hypocotyls. CtFAD2-5 and -8 appear to be root-specific and CtFAD2-10 is mostly expressed

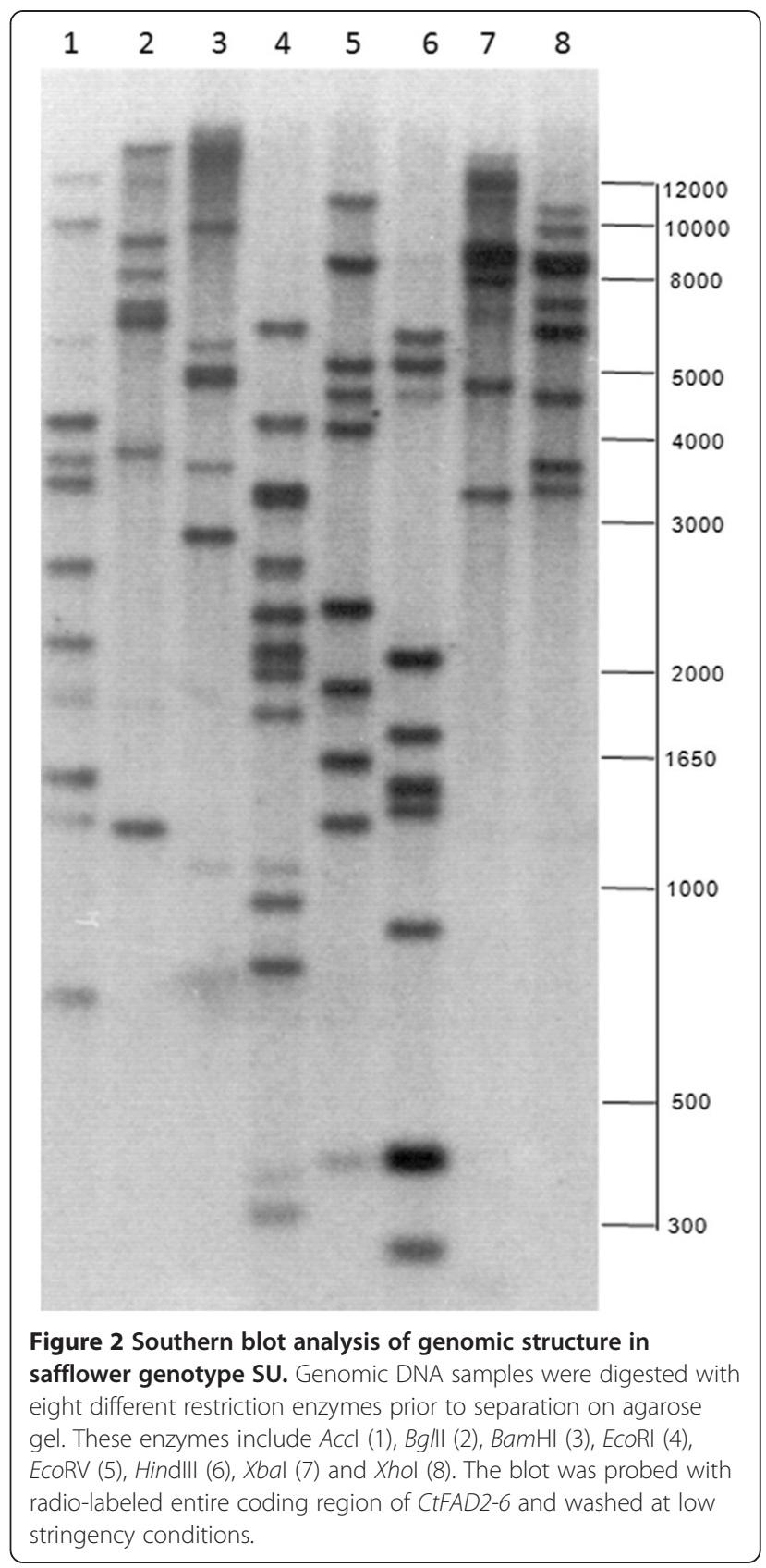

in flower tissues, with relatively low levels detected in other tissues.

\section{Expression of safflower CtFAD2 genes in Saccharomyces cerevisiae}

Expression in yeast (Saccharomyces cerevisiae) cells has been successfully used for studying the functional properties of several plant FAD2 $\Delta 12$ oleate fatty acid desaturases $[19,48,49]$ because it has a simple fatty acid profile, contains ample oleic acid that can serve as precursor for FAD2 enzymes, and lacks an endogenous FAD2 activity. As shown in Figure 4, polyunsaturated 


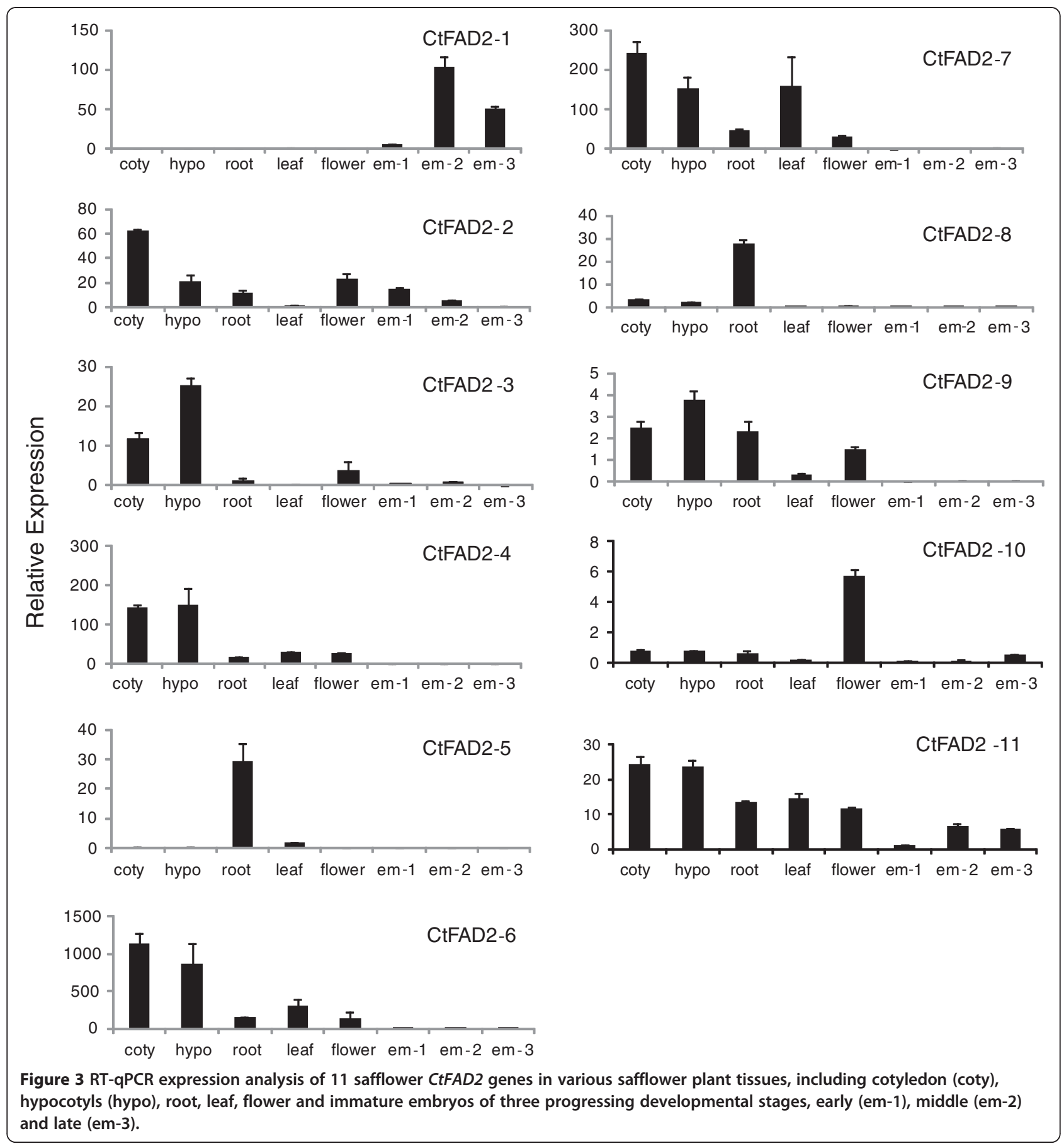

fatty acids including C16:2 and C18:2 were not produced in the control yeast cells transformed with the empty pYES2 vector. By reference to the standard gas chromatogram of C16:2 and C18:2 fatty acid methyl esters (FAMEs) having retention time of $8.513 \mathrm{~min}$ and 11.293 min, respectively, C18:2 was found to be present in the yeast lines expressing CtFAD2-1 (Figure 4B), CtFAD2-2 (Figure 4C), CtFAD2-10 (Figure 4E), and C16:2 was seen in yeast lines expressing CtFAD2-9 (Figure 4D) and
CtFAD2-10 (Figure 4E). This result indicated that CtFAD2-1, CtFAD2-2 and CtFAD2-10 are $\Delta 12$ oleate desaturases that convert oleic acid to linoleic acid. It appears that CtFAD2-9 is a $\Delta 12$ palmitoleate desaturase that prefers $\mathrm{C} 16: 1$ to $\mathrm{C} 18: 1$ substrate, leading to specific production of $\mathrm{C} 16: 2$. As shown in Figure $4 \mathrm{~F}$ and Figure 4G, the expression of CtFAD2-11 resulted in two minor, yet distinct peaks, at retention time of 10.642 min and 11.293 min, respectively. The latter peak is 


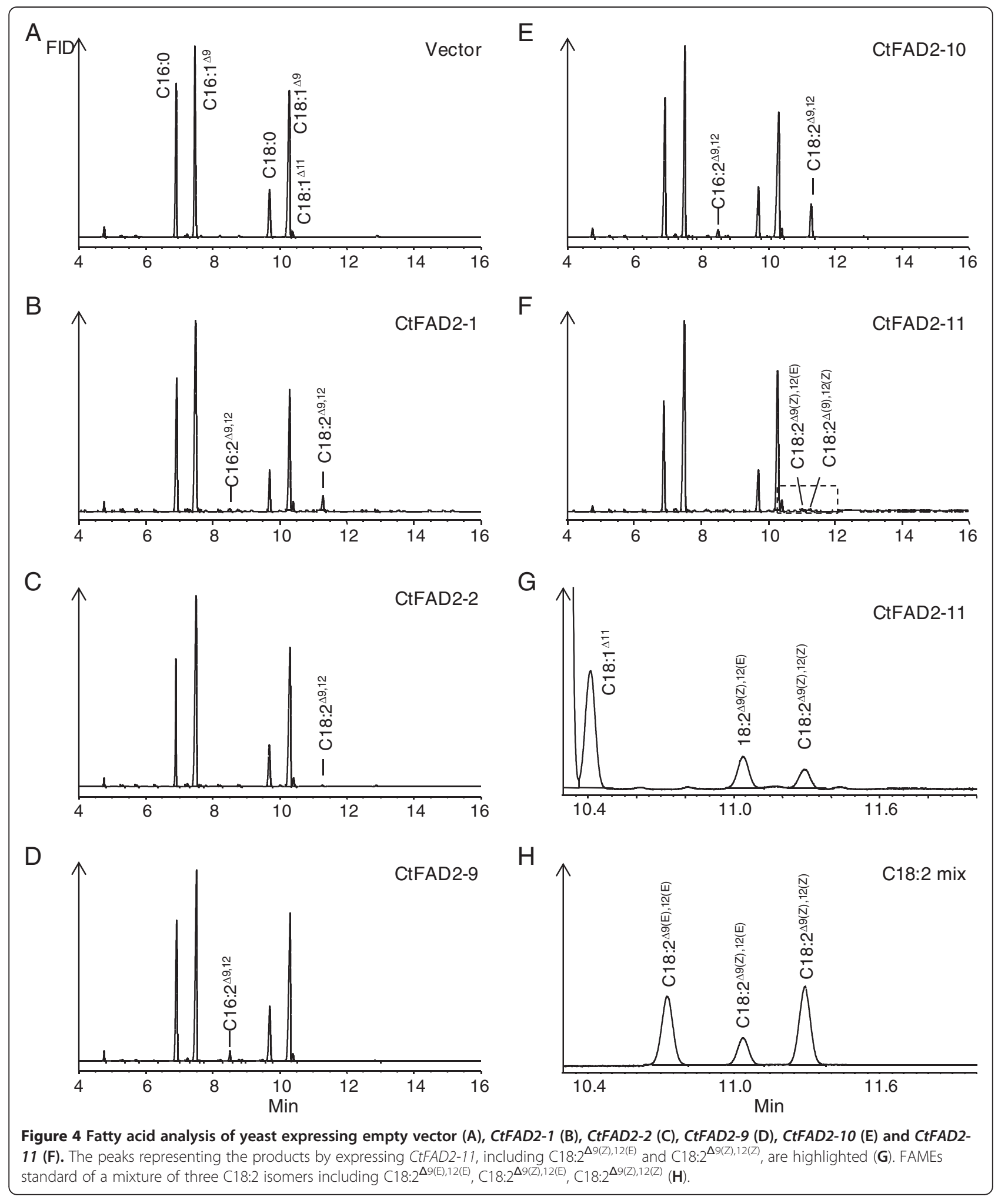


Table 3 Fatty acid composition of yeast cells expressing selected CtFAD2 genes

\begin{tabular}{|c|c|c|c|c|c|c|}
\hline & vector & CtFAD2-1 & CtFAD2-2 & CtFAD2-9 & CtFAD2-10 & CtFAD2-11 \\
\hline C14:0 & $1.3 \pm 0.15$ & $1.2 \pm 0.02$ & $1.2 \pm 0.06$ & $1.1 \pm 0.06$ & $1.0 \pm 0.01$ & $0.6 \pm 0.01$ \\
\hline C14:1 & $0.3 \pm 0.06$ & $0.3 \pm 0.01$ & $0.3 \pm 0.01$ & $0.2 \pm 0.01$ & $0.3 \pm 0.02$ & $0.2 \pm 0.00$ \\
\hline C16:0 & $23.6 \pm 1.62$ & $23.0 \pm 0.04$ & $22.0 \pm 0.46$ & $21.3 \pm 0.59$ & $22.3 \pm 0.03$ & $18.4 \pm 0.34$ \\
\hline C16:1 & $36.0 \pm 1.77$ & $37.2 \pm 0.16$ & $36.6 \pm 0.07$ & $34.3 \pm 0.54$ & $34.8 \pm 0.21$ & $37.3 \pm 0.16$ \\
\hline C16:2 & 0.0 & $0.3 \pm 0.02$ & 0.0 & $1.6 \pm 0.09$ & $1.2 \pm 0.03$ & 0.0 \\
\hline C18:0 & $7.7 \pm 0.74$ & $7.4 \pm 0.12$ & $7.4 \pm 0.07$ & $8.9 \pm 0.13$ & $8.1 \pm 0.09$ & $7.5 \pm 0.07$ \\
\hline C18:1 & $29.6 \pm 0.99$ & $26.4 \pm 0.24$ & $31.0 \pm 0.51$ & $31.3 \pm 0.25$ & $25.4 \pm 0.07$ & $33.3 \pm 0.26$ \\
\hline$C 18: 1^{\Delta 11}$ & $1.4 \pm 0.20$ & $1.6 \pm 0.02$ & $1.5 \pm 0.04$ & $1.3 \pm 0.08$ & $1.3 \pm 0.01$ & $1.9 \pm 0.01$ \\
\hline C18:2 & 0.0 & $2.8 \pm 0.14$ & $0.1 \pm 0.11$ & 0.0 & $5.5 \pm 0.09$ & $0.3 \pm 0.02$ \\
\hline $\mathrm{C} 18: 2^{\Delta 9(\mathrm{Z}), 12(\mathrm{E})}$ & 0.0 & 0.0 & 0.0 & 0.0 & 0.0 & $0.5 \pm 0.03$ \\
\hline$n=3$ & & & & & & \\
\hline
\end{tabular}

corresponding to FAME of linoleic acid $\left(18: 2^{\Delta 9(\mathrm{Z}), 12(\mathrm{Z})}\right)$, and the former peak was identified as FAME of its $\Delta 12$ trans isomer $\left(\mathrm{C} 18: 2^{\Delta 9(\mathrm{Z}), 12(\mathrm{E})}\right)$ by GC-MS of their pyrrolidide adducts, and 2,4-dimethyloxazoline (DMOX) modification (Additional file 1: Figure S2). The fatty acid composition of yeast cells expressing CtFAD2-1, -2, -9, -10 and -11 is shown in Table 3 . As shown in the Table 3, the expression of CtFAD2-9 produced 1.6\% C16:2, without any C18:2. No new peaks were detected in yeast cells expressing CtFAD2-3, $-4,-5,-6,-7$, and -8 .

To examine whether any CtFAD2 have fatty acid hydroxylase activity, FAMEs prepared from the yeast cells expressing each of the 11 CtFAD2 genes were reacted with a silyating reagent that can convert hydroxyl residues into TMS-ether derivatives from which the mass spectrum can be examined. However, none of hydroxyl derivatives of common fatty acids, such as ricinoleic acid synthesised from oleic acid, was detected in any of the yeast cell lines expressing CtFAD2 genes, indicating the absence of fatty acid hydroxylase activity in the CtFAD2 enzymes. Further tests for potential epoxygenase and acetylenase activities that use linoleic acid as substrate have also been carried out in these transgenic yeast cell lines, by supplementing free linoleic acid in the yeast culture upon the induction by galactose. No novel peaks representing epoxy or acetylenic fatty acids were detected (data not presented).

\section{Transient expression in $N$. benthamiana leaves}

The function of CtFAD2-11 was initially assessed by expression in $S$. cerevisiae, and two novel fatty acids were identified by GC-MS as $18: 2^{\Delta 9(\mathrm{Z}), 12(\mathrm{Z})}$ and $18: 2^{\Delta 9}$ (Z),12(E) respectively. Consistent with the results obtained from yeast, constitutive expression of CtFAD2-11 driven by $35 \mathrm{~S}$ CaMV promoter in $N$. benthamiana leaves, co-expressed with 35S:P19 yielded a novel product that is not present in $N$. benthamiana leaf when infiltrated with 35S:P19 alone (Figure 5A). The methyl ester of this
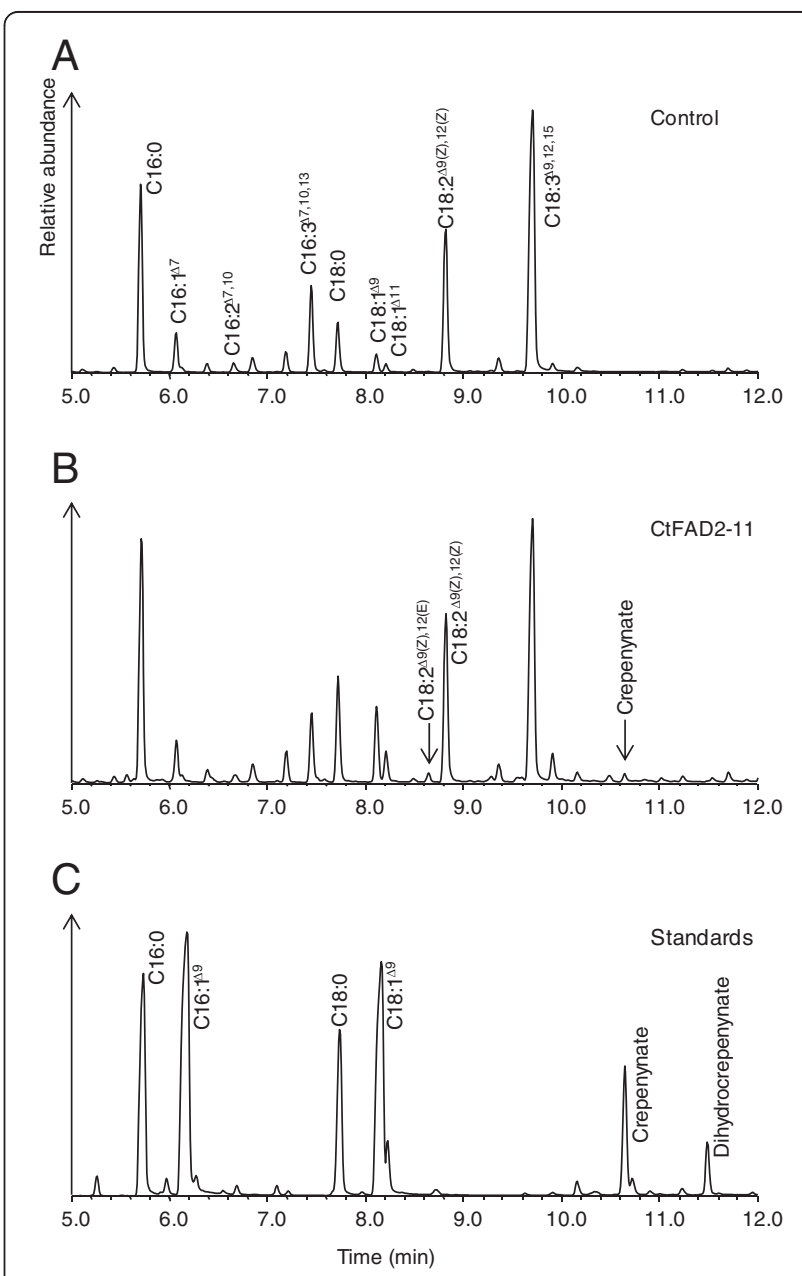

Figure 5 Fatty acid analysis of $N$. benthamiana leaves transiently expressing CtFAD2-11. (A) negative control expressing 35S:P19 alone. (B) co-expression of 35S:P19 and 35S:CtFAD2-11, showing two novel peaks representing the products of CtFAD2-11, C18:2 $2^{\Delta 9(\mathrm{Z}), 12(\mathrm{E})}$ and crepenynic acid. (C) fatty acid standard, including the crepenynic acid. 
Table 4 Fatty acid composition of $N$. benthamiana leaves transiently expressing CtFAD2-11

\begin{tabular}{lll}
\hline & Control & CtFAD2-11 \\
\hline $\mathrm{C} 16: 0$ & $17.4 \pm 0.48$ & $23.7 \pm 2.57$ \\
$\mathrm{C} 16: 1$ & $0.3 \pm 0.22$ & $0.3 \pm 0.05$ \\
$\mathrm{C} 16: 2$ & $0.8 \pm 0.12$ & $0.6 \pm 0.09$ \\
$\mathrm{C} 16: 3$ & $7.2 \pm 0.15$ & $5.5 \pm 0.81$ \\
$\mathrm{C} 18: 0$ & $3.3 \pm 0.33$ & $5.3 \pm 0.72$ \\
$\mathrm{C} 18: 1^{\Delta 9}$ & $1.0 \pm 0.09$ & $3.8 \pm 0.30$ \\
$\mathrm{C} 18: 1^{\Delta 11}$ & $0.5 \pm 0.33$ & $1.2 \pm 0.36$ \\
$\mathrm{C} 18: 2^{\Delta 9(\mathrm{Z}), 12(\mathrm{E})}$ & 0.0 & $0.4 \pm 0.07$ \\
$\mathrm{C} 18: 2^{2}$ & $12.0 \pm 0.65$ & $11.6 \pm 0.84$ \\
$\mathrm{C} 18: 3^{2}$ & $56.8 \pm 0.19$ & $45.8 \pm 4.01$ \\
$\mathrm{C} 20: 0$ & $0.5 \pm 0.10$ & $1.0 \pm 0.19$ \\
$\mathrm{C} 20: 1^{\mathrm{Ac}}$ & $0.2 \pm 0.15$ & $0.4 \pm 0.04$ \\
$\mathrm{C} 18: 2^{\mathrm{AC}}$ & 0.0 & $0.5 \pm 0.06$ \\
$\mathrm{n}=3$ & & \\
\hline
\end{tabular}

new product displayed a GC retention time (8.642 Min) that was identical to that of a methyl $18: 2^{\Delta 9(\mathrm{Z}), 12(\mathrm{E})}$ (Figure 5B). The novel 18:2 $2^{\Delta 9(\mathrm{Z}), 12(\mathrm{E})}$ accounted for $0.4 \%$ of the fatty acids of leaves transiently expressing CtFAD2-11 (Table 4). In addition, another new peak that was not observed in the yeast cultures was detected at retention time of 10.642 min (Figure 5B). The total ion chromatogram and mass spectrum of this new fatty acid were consistent with that of crepenynic acid (9-octadecen12-ynoic acid) (Additional file 1: Figure S3), demonstrating that the CtFAD2-11 polypeptide had $\Delta 12$-acetylenase activity as suggested by its close sequence alignments with known $\Delta 12$ fatty acid acetylenases. As shown in Table 4, crepenynic acid accounts for $0.5 \%$ of total fatty acids in $N$. benthamiana leaves expressing CtFAD2-11.

It is notable that the expression of CtFAD2-11 transiently in the $N$. benthamiana cells resulted in a significant increase of saturated and monounsaturated fatty acids at the expense of polyunsaturated fatty acids including linoleic acid and $\alpha$-linolenic acid (ALA), relative to the untransformed control (Table 4). Overall, the results from the yeast and $N$. benthamiana expression experiments indicated that CtFAD2-11 functioned primarily as a $\Delta 12$ oleate desaturase lacking stereo-specificity, producing both linoleic acid and its trans- $\Delta 12$ isomer. In addition, it could also further desaturate the $\Delta 12$ double bond of linoleic acid to form the acetylenic bond of crepenynic acid.

The other 10 CtFAD2 genes have also been expressed in $N$. benthamiana leaves, but we did not observe production of any additional novel fatty acids compared to the controls (data not presented).

\section{Discussion}

We report here the cloning of 11 distinct safflower CtFAD2 genes, which does not include at least three more partial severely truncated EST sequences that are also present in the CPG safflower EST database. Furthermore, due to the current lack of safflower genomics information it remains unclear whether there are additional unexpressed FAD2 members. Taken together, this is to our knowledge the largest FAD2 gene family in any species examined to date. In spite of their high conservation of putative amino acid sequences, individual CtFAD2 genes have clear distinguishing features, especially with respect to their $\mathrm{N}$ - and C-terminal ends, $5^{\prime}$ and $3^{\prime}$ UTR regions, and the size, location and sequence of an intron located at the $5^{\prime}$ UTR.

FAD2 is among the best-studied plant fatty acid desaturase gene families, in terms of both molecular and biochemical investigations. Although only a single representative was identified in Arabidopsis [11], FAD2 appears to exist as complex gene families in most other plant genomes studied so far. Two distinct FAD2 genes have been described in soybean [29], flax [50,51] and olive [49]; three genes in sunflower [52] and Camelina sativa [53]; and four genes in cotton [31-34]. In the amphitetraploid Brassica napus, 4-6 different FAD2 genes have been identified in each diploid sub-genome [30].

Although comparable studies are lacking, it seems that safflower is unusual with respect to $F A D 2$ gene family evolution. Safflower is a self-pollinating diploid plant species that is most closely related to a wild diploid species C. palaestinus and extensive genome duplication or re-arrangement has not previously been reported in safflower [54]. In the absence of genomic data, it is not known whether the significant amplification in gene copy number observed for FAD2 also occurs for other gene families in safflower. However, our own limited observations of other safflower lipid biosynthesis genes do not show evidence for such expansive gene family size (unpublished data).

The complex FAD2 family cannot be attributed to alternative splicing as $\mathrm{Fad} 2$ genes do not contain introns in the coding region sequence. Instead, gene duplication is more likely responsible for creating the FAD2 family complexity in safflower. Topology of the gene tree show that gene duplications have probably occurred at several hierarchical levels, i.e. at various times during evolution. For example, the CtFAD2-3, $-4,-5$ are more closely related to each other than they are to other safflower $F A D 2$ sequences, indicating that more recent gene duplications are responsible for the emergence of this clade. It is not known how many FAD2 genes were generated after the speciation of safflower, in other words, are unique to the $C$. tinctorius species. Analysis of FAD2 genes from taxonomically closely related plant species, such as those from the genus Centaurea within the 
Asteraceae family, could perhaps provide a better picture of exactly when the duplication events occurred.

Genetic redundancy is a prime feature of plant genomes, providing the opportunity for additional divergent functions to evolve while retaining the original function of vital genes. To persist over long periods of evolutionary time, new gene duplications must be positively selected for, otherwise they can degenerate to pseudogenes by accumulating nonsense mutations, frame shifts and even insertion/deletion events. Once becoming a pseudogene, the likely fate is to continue to accumulate mutations at the maximum rate and become increasingly divergent. The fact that all the $11 C t F A D 2$ genes are expressed and translated is evidence that at least these duplicated $F A D 2$ genes are positively selected in safflower. As the current research focused on the expressed FAD2 genes, it is plausible that other duplicated FAD2 genes might have lost expression and functionality through gene pseudogenization or gene deletion.

The production of linoleic acid through the $\Delta 12$ desaturation catalyzed by FAD2 is central to the functionality of biological membrane systems, cellular signalling, thermal adaptation, and energy storage [55]. When plants are subject to adverse environmental conditions, a wide range of cellular response occurs, including the adjustments of unsaturation levels of membrane fatty acids. Plant membrane integrity and function, determined by structure and fluidity, are significantly affected by lipid composition, particularly the degree of fatty acid desaturation [23]. Abiotic environmental stresses such as cold, heat, drought and salt are able to induce changes in fatty acid composition. Expression of FAD2 is also regulated by adverse environmental factors, suggesting the possible involvement of FAD2 in plant response to abiotic stress [24]. During recent years a number of FAD2-divergent genes have been identified in other members of the Asteraceae family that safflower belongs to, such as marigold (Calendula officinalis), Crepis alpina and sunflower (Helianthus annuus) [15,39,56] and have been associated with synthesis of divergent fatty acid structures that may play roles in resistance to biotic stresses. For example, the epoxygenated fatty acids were found to be potent inhibitors for the germination and enlargement of spores of rice blast fungus [57]. Other unusual fatty acids, acetylenic fatty acids, are involved in the biosynthesis of polyacetylenes that act as phytoalexins in numerous Asteraceae species including safflower and therefore play a significant role in plant disease resistance [58-60].

It is intriguing how the divergent FAD2 gene family members with similar fundamental properties carry out specific functions. The intrinsic characteristics of various FAD2 genes, as exemplified by some conserved motifs, are likely determining their distinct expression patterns and abilities to respond to environmental stimuli, and diversified functionalities. It has been demonstrated through site directed mutagenesis that very few amino acid changes are required to change the enzymatic function of a $F A D 2$ gene. For instance, as few as four amino acid changes in a FAD2 fatty acid desaturase were required in order to obtain hydroxylase activity, and conversely, substitution of six amino acids could convert a fatty acid hydroxylase into a fatty acid desaturase [21]. The $\Delta 12$ acetylenase from Crepis alpina deviates from $\Delta 12$ desaturase in 29 positions [15], but it has not been determined how many of these changes are required to facilitate the functionality alteration. It is suggested that a switch from desaturase to acetylenase might involve more extensive changes in sequence than that required to interchange between a fatty acid desaturase and a fatty acid hydroxylase. The origins of specificity leading to acetylenases and desaturases are not currently evident from comparisons at the primary sequence level, and residues promoting acetylenase activity have yet to be located. Interestingly, a Gly replaces Ala at the amino acid immediate preceding the first His box in all the known acetylenases, in contrast to a typical fatty acid desaturase or hydroxylase. It is suggested that this residue may facilitate acetylenase chemistry by alleviating steric hindrance by providing substrate pocket spatial flexibility to permit the conversion of a "kinked" substrate (cis-ene) into a straight chain product (acetylene) [61].

We have identified that, although a large functional $F A D 2$ gene family exists in safflower, only FAD2-1 has exclusively high expression in developing safflower seeds. It appears that this gene plays the major role in producing the very high levels of linoleic acid present in seed storage oils of the wild-type (high-linoleic) safflower. FAD2-2, the presumed house-keeping microsomal $\Delta 12$ oleate desaturase has a generally constitutive expression throughout the plant. It is interesting to note that in this study we have found that CtFAD2-2 has significantly higher expressioin in cotyledonary tissues of a young seedling, compared to all other safflower tissues examined. Although likely contributing to the overall oleate desaturase activity in safflower vegetative tissues (where Fad6 also plays a significant role), CtFAD2-2 presumably plays only a secondary role in linoleic acid production in the seed oil.

The expression of CtFAD2-9 in yeast led to the specific synthesis of palmitolinoleic acid $\left(\mathrm{C} 16: 2^{\Delta 9,12}\right)$, presumably produced by the desaturation of palmitoleic acid. It is well established that the initial desaturation of a C16 saturated fatty acid, palmitic acid occurs in the plastids by the action of the stearoyl-ACP $\Delta 9$-desaturase to form palmitoleic acid that could be exported to the cytosol and incorporated into phospholipids for further desaturation by a FAD2 oleate $\Delta 12$-desaturase [12]. To 
our knowledge, the safflower CtFAD2-9 is the first described FAD2 enzyme specific for the production of palmitolinoleic acid. Palmitolinoleic acid does not normally accumulate in abundance in plant tissues as it is quickly converted to hexadecatrienoic acid (C16:3) by abundant $\Delta 15$-desaturase in vegetative tissues, and in seed tissue there is limited availability of C16:1 substrate. In olive leaves and mesocarp tissues, increased accumulation of palmitolinoleic acid was observed in respond to wounding [62]. It was suggested that the increase of palmitolinoleic acid in wounded olive mesocarp is a result of enhanced demand for substrates for the synthesis in the chloroplast of hexadecatrienoic acid that could be used as precursors via the lipoxygenase pathway of signal molecules involved in plant defence and wounding [63]. However, the involvement of C16 polyunsaturated fatty acids in the oxylipin biosynthesis is less documented compared to its $\mathrm{C} 18$ counterparts. It remains to be revealed what the specific evolutionary drive is for CtFAD2-9 that specifically produces palmitolinoleic acid in safflower.

Among the 11 safflower CtFAD2 genes, CtFAD2-10 is the first characterised $F A D 2$ gene with preferential expression in safflower heads prior to seed set. However this is similar to the situation in cotton where of the four different genes encoding FAD2 isolated one member, ghFAD2-1, was specifically expressed in both developing flower buds and seeds [33]. It is known that jasmonic acid derived from ALA plays a significant role as a chemical signal controlling stamen and pollen development, especially in the final stages of pollen development and anther dehiscence in Arabidopsis [28]. As the precursor of ALA, therefore, the enhanced production of linoleic acid by FAD2 may play an essential role in flower development. It is likely that the retention of CtFAD2-1 as a strong $\triangle 12$-desaturase for synthesis of seed storage lipids has freed up the duplicated CtFAD210 for diversification into a specialised flower-specific form. Evolutionary modifications of the CtFAD2-10 gene that favoured flower expression at the expense of seed expression would be unlikely to have any negative fitness consequences given the retention of CtFAD2-1 seed expression and the relatively inconsequential impact that moderate variations in oleic-linoleic ratios in seed lipids would be expected to have on fitness.

We demonstrated that the CtFAD2-11 $\Delta 12$ acetylenase is a tri-functional enzyme that could desaturate both oleate and linoleate. It produces a mixture of $\Delta 12(\mathrm{Z})$ and (E) isomers of C18:2 from oleate in yeast cells. In plant cells, CtFAD2-11 could also produce crepenynic acid (C18:2 ${ }^{\Delta 9(\mathrm{Z})}$,-octadecen-12-ynoate) from linoleate, as demonstrated by transient expression in a $N$. benthamiana leaf. Such a multi-functionality has previously been reported in Crepis alpina where CREP1 could produce both cis and trans isomers of C18:2 fatty acid, in addition to producing crepenynic acid in transgenic Arabidopsis [22]. When expressed in yeast, CtFAD2-11 produces a higher level of $\mathrm{C} 18: 2^{\Delta 9(\mathrm{Z}), 12(\mathrm{E})}$ than linoleic acid, consistent with the expression of CREP1 in Arabidopsis seeds [22]. C18: $2^{\Delta 9(\mathrm{Z}), 12(\mathrm{E})}$ has been identified as an apparent metabolic dead end as it cannot be converted further into crepenynic acid. The cis and trans FAD2-mediated $\Delta 12$ oleate desaturation likely have very similar catalytic mechanisms but differ in substrate binding properties. Compared to a normal FAD2 oleate cis- $\Delta 12$ desaturase, CtFAD2-11 might have an altered substrate interaction geometry at the active site. A less restrictive binding pocket could allow a small rotation of the substrate and permit the formation of an $E / Z$ mixture. It was previously demonstrated that a $\mathrm{C} 18: 2^{\Delta 9(\mathrm{Z}), 12(\mathrm{Z})}$ is produced by abstraction of the pro- $\mathrm{R}$ hydrogens at $\mathrm{C} 12$ and $\mathrm{C} 13$, whereas and $C 18: 2^{\Delta 9(\mathrm{Z}), 12(\mathrm{E})}$ is produced by scission of the (12R,13S) hydrogens [22].

It appears that the $\Delta 12$ trans-double bond is a co-product of acetylenic fatty acid production by a specialised FAD2 enzyme in both safflower and Crepis. The trans desaturation of oleate to form $\mathrm{C} 18: 2^{\Delta 9(\mathrm{Z}), 12(\mathrm{E})}$ has also been shown by another functionally divergent FAD2 enzyme, the $\Delta 12$ fatty acid conjugase from tung (Aleurites fordii), which displayed trans- $\Delta 12$ oleic acid desaturase activity, in addition to its conjugase activity [19]. The production of a trans double bond, accompanied by the production of other unusual fatty acids, such as calendic acid (C18:3 $3^{8(\mathrm{E}), 10(\mathrm{E}), 12(\mathrm{Z})}$ ) in Calendula, and dimorphecolic acid (9OH-18:2 $\left.2^{\Delta 10(\mathrm{E}), 12(\mathrm{E})}\right)$ in Dimorphotheca sinuate by divergent FAD2 fatty acid modifying enzymes has also been reported $[20,45,64]$.

In the transient expression system of $N$. benthamiana leaves, we have observed that the expression of CtFAD211 resulted in an increase in the relative proportions of saturated and monoenoic fatty acids at the expense of polyunsaturated fatty acids. This is consistent with what has previously been observed in the expression of acetylenases and epoxygenases in Arabidopsis and cotton $[65,66]$. The production of vernolic acid, by modest seed-specific expression of the $C$. palaestina $\Delta 12$-epoxygenase ( $C$ pal2) gene in Arabidopsis and cotton seeds led to enhanced oleic acid accumulation at the expense of linoleic acid compared to the wild type seeds. The subsequent co-expression of $C$. palaestina $\Delta 12$ desaturase (Cpdes) with Cpal2 was shown to restore the normal oleate desaturation and therefore further raised the vernolic acid level. This highlights that substrate availability is one factor controlling diverged FAD2 desaturase activity. Potential alternative mechanisms for the reduced levels of linoleic acid in $N$. benthamiana leaves expressing the CtFAD2-11 gene could include a degree of homology-based post-transcriptional silencing of $\Delta 12$ - 
desaturase members of the FAD2 gene family that are operational in the leaf, or the possible formation of potentially ineffective heterodimers between CtFAD2-11 and the FAD2 $\Delta 12$-desaturase proteins [67].

Based on sequence alignments CtFAD2-11 is most closely related to the sunflower vFAD2 that is characterized as a fungal elicitor-inducible $\Delta 12$ fatty acid acetylenase and could produce both crepenynate and (14Z)-dehydrocrepenynate when expressed in developing soybean somatic embryos [39]. Divergent FAD2 genes with the same catalytic functionality as the sunflower $v F A D 2$ have also been isolated from numerous plant species, including the EIl12 from parsley (Petroselinum crispum L.), C. officinalis and English ivy (Hedera helix L.) [39]. Although sharing higher sequence homology to vFAD2 than C. alpina CREP1, when expressed in $N$. benthamiana CtFAD2-11 was shown to have the same functionality as CREP1, it did not have the additional synthesis of (14Z)-dehydrocrepenynic acid typical of the $v F A D 2$-like genes.

Accumulation of acetylenic fatty acids or any other unusual fatty acids that would be synthesized by divergent forms of FAD2 enzymes was not observed in various tissues of safflower, consistent with previous findings with $C$. alpina and $H$. annuus flower tissue even though gene expression was detected $[39,68]$. Identifying the function and control mechanisms of such cryptic expression of unusual fatty acids is a poorly explored aspect of fatty acid metabolism. It is likely that acetylenic fatty acids are synthesized in low amounts and are rapidly metabolized for the formation of secondary bioactive molecules. Crepenynic and dehydrocrepenynic acids are believed to be intermediates in the biosynthetic pathway of biologically active polyacetylenic compounds that are known to occur in safflower as well as some other members of the Asteraceae, Apiaceae, and Araliaceae families $[59,60,69,70]$. However, many of the genes necessary for the formation of the putative antifungal polyacetylenes need to be discovered and the in vivo functions of functionally divergent FAD2(s) in the polyacetylene pathway remain to be established.

In addition to the above mentioned safflower FAD2 genes, another six CtFAD2 genes, from CtFAD2-3 through to $C t F A D 2-8$, are highly expressed in rapidly expanding tissues such as cotyledons, hypocotyls, and roots in the young safflower seedlings. It is not apparent why multiple FAD2 genes would be expressed in young seedling tissues in safflower, although enhanced FAD2 expression and increased linoleic acid production was previously observed in sunflower cotyledons immediately upon germination [52,71]. However, these CtFAD2 genes are possibly more likely to be performing reactions other than oleate desaturases, considering that their primary sequence features are more closely associated with functionally divergent FAD2 homologs and they were unable to synthesise linoleic acid when expressed in yeast.

\section{Conclusions}

The identification and initial characterization of the 11 full length FAD2 cDNAs and their corresponding structural genes provides an insight into the principal determinants of synthesis of linoleic acid in safflower seed oil. It also presents an unprecedented opportunity to fully understand the fundamental functions and diversity of FAD2 proteins and their differing fatty acid modifications within a single plant species. Characterization of the biochemical properties of each $F A D 2$ gene will lend further insight into their potential roles. Identifying the specific structural attributes of the diverged FAD2 enzymes that control their regio- and chemo- selectivity would be greatly accelerated by availability of a structure-function characterization of the microsomal fatty acid desaturases, an elusive goal that would have a wide ranging impact on lipid metabolic research. Defining the phenotype of transgenic plants with RNAi down regulation of each $F A D 2$ gene will contribute to the clarification of the pathways attributed by FAD2 members. Ultimately, the use of divergent FAD2 enzymes to produce novel fatty acids, such as crepenynic acid, in genetically engineered crop plants may not only provide opportunities for protection from disease or pests to the plant but also allow for the synthesis of economically valuable oleochemical products.

\section{Methods}

\section{Plant materials and growth conditions}

Seed of wild type (high-linoleic) safflower (Carthamus tinctorius L.) was sourced from a commercial birdseed supplier in Australia and was designated as genotype SU. SU safflower plants were grown from seed in the glasshouse in a perlite and sandy loam potting mix under a day/night cycle of $16 \mathrm{hrs}\left(25^{\circ} \mathrm{C}\right) / 8 \mathrm{hrs}\left(22^{\circ} \mathrm{C}\right)$. Plant tissues including leaves, roots, cotyledons and hypocotyls were sampled from young germinating safflower seedlings. Flowering heads were obtained at the first day of flowering and developing embryos were harvested at three stages representing early, middle and late seed development. Samples were immediately chilled in liquid nitrogen and stored at $-80^{\circ} \mathrm{C}$ until DNA and RNA extraction.

\section{Total RNA extraction and CDNA synthesis}

Total RNA isolation was performed from $100 \mathrm{mg}$ of frozen safflower tissues using RNeasy ${ }^{\circledR}$ Plant total RNA kit (Qiagen, Hilden, Germany) as described by manufacturer's protocol. RNA concentration was determined by NanoDrop $^{\mathrm{TM}}$ spectrophotometer ND1000 (Thermo Fisher Scientific, Victoria, Australia) and concentrations were equalized before analysis. Quality and relative quantities 
were also visualized by standard RNA formaldehyde agarose gel $(1 \%, w / v)$. Total RNA was then treated with RQ1 RNase-free DNase (Qiagen, Hilden, Germany) to remove contaminating genomic DNA. First-strand cDNA was synthesized from 400 ng of DNA-free total RNA using the SuperScript III First-Strand Synthesis System (Qiagen, Hilden, Germany) with oligo $(\mathrm{dT})_{20}$ primer, following the manufacturer's instructions.

\section{Construction and screening of cDNA library derived from safflower developing embryos}

To isolate FAD2 cDNAs a lambda cDNA library was constructed using safflower developing embryos. A mixture of immature embryos of different developmental stages were harvested and ground to powder in liquid nitrogen and RNA extraction was carried out using TRIzol following the manufacturer's instruction (Invitrogen, Carlsbad, CA, USA). Poly(A)-containing RNA was isolated using Qiagen mRNA purification kit (Qiagen, Hilden, Germany). First strand oligo(dT)-primed cDNA was synthesised and converted to double stranded using Stratagene cDNA synthesis kit, according to the manufacturer's instructions (Stratagen, La Jolla, CA, USA). The blunt-ended cDNA was ligated with EcoRI adaptors, phosphorylated, and size fractionated by gel-filtration in a Chroma spin+TE-400 column (Clontech, CA, USA). The recombinants were propagated in the $E$. coli strain XL-1 Blue MRF' using a Stratagene Predigested $\lambda \mathrm{ZAP}$ II/EcoRI/ CIAP cloning kit (Invitrogen, Carlsbad, CA, USA).

To identify the FAD2 cDNAs, the cDNA library was screened using a DNA fragment corresponding to the coding region of Arabidopsis FAD2 following the protocol previously described [31]. Positive plaques were purified through two successive rounds of screening and the purified phagemids containing putative FAD2 cDNAs were excised as outlined in the Stratagene $\lambda \mathrm{ZAPII}$ cDNA Synthesis Kit instruction manual (Invitrogen, Carlsbad, CA, USA). Sequence analysis of the FAD2 sequences were identified by the NCBI Blast program (http://www. ncbi.nlm.nih.gov/BLAST/). The open reading frame was predicted by using Vector NTI.

\section{Isolation of the full length and the open reading frame (ORF) of FAD2 genes expressed in non-seed tissues}

We have also interrogated the Composite Genome Project (CGP) expressed sequence tag (EST) database of safflower (http://cgpdb.ucdavis.edu/cgpdb2.), which was screened by "Blast" for similarity with Arabidopsis FAD2 sequence (AT3G12120). Putative safflower FAD2 ESTs were compared and the longest EST clones of the strongest hits from different contigs were selected for extension into full length by performing $3^{\prime}$ Rapid Amplification of cDNA Ends (RACE) using Bioline one step RT-PCR kit following Manufacturers' instructions (Bioline, London, UK). A gene-specific primer (GSP) was used for each of the selected ESTs, in combination with a poly $(\mathrm{dT})$ primer with a NotI site at its $3^{\prime}$ end. A second round of PCR was performed using a nested GSP in combination with the poly (dT) primer. GSPs for $3^{\prime}$ RACE were listed in Additional file 1: Table S1. The one-step reverse transcriptase PCR reactions were performed using 200 ng of RNA as templates starting with an initial reverse transcription reaction at $50^{\circ} \mathrm{C}$ for $30 \mathrm{~min}$, followed by $95^{\circ} \mathrm{C}$ for $3 \mathrm{~min}$, and 40 cycles at $95^{\circ} \mathrm{C}$ for $30 \mathrm{~s}, 55^{\circ} \mathrm{C}$ for $30 \mathrm{~s}$, and $72^{\circ} \mathrm{C}$ for $1 \mathrm{~min}$, and a final extension at $72^{\circ} \mathrm{C}$ for 10 mins. Cloning of the $5^{\prime}$ end of the CtFAD2-6 cDNA was performed with $5^{\prime}$ RACE System Kit (Invitrogen, Carlsbad, CA, USA) following manufacturers' instruction. Only the CtFAD2-6 mRNA was reverse transcribed to cDNA using a genespecific primer GSP1, 5'- ACCTAACGACAGTCATGA ACAAG - $3^{\prime}$. A gene-specific primer GSP2, 5'- GTGAGG AAAGCGGAGTGGACAAC $-3^{\prime}$ was used in the first PCR amplification. A hot start at $95^{\circ} \mathrm{C}$ for 4 min before adding the polymerase, 33 cycles of denaturation at $94^{\circ} \mathrm{C}$ for $45 \mathrm{~s}$, annealing at $55^{\circ} \mathrm{C}$ for $1 \mathrm{~min}$ and extension at $72^{\circ} \mathrm{C}$ for 2 min. The amplification products were fractionated on $1 \%$ agarose gel from which the DNA fragments with expected sizes were purified and subcloned into the vector pGEMTeasy $^{\circledR}$ (Promega, Madison, WI, USA) and identity confirmed by DNA sequencing using an ABI 373 sequencer.

The ORFs of FAD2 genes were amplified using the same One-step RT-PCR kit (Bioline, London, UK) on total RNAs derived from several safflower plant tissues and PfuUltra II Fusion HS DNA Polymerase (Stratagen, La Jolla, CA, USA). The primers (Additional file 1: Table S2) used to amplify the ORFs were designed based on the DNA sequences located in the $5^{\prime}$ and $3^{\prime}$ UTR of each gene. The amplified PCR products were cloned to vector pGEM-Teasy $^{\circledR}$ (Promega, Madison, WI, USA), and verified by DNA sequencing. The cloned amplification products were addressed with the gene name FAD2 and a suffix representing the first letter of the genus ( $C$ for Carthamus) and species ( $\mathrm{t}$ for tinctorius).

\section{DNA isolation and Southern blot analysis}

The genomic DNA of safflower genotype SU was isolated from fully expanded leaves using CTAB buffer following the method described by Paterson et al. (1993). Further purification was carried out using $\mathrm{CsCl}$ gradient as previously described [31]. About $10 \mu \mathrm{g}$ safflower genomic DNA was digested with seven different restriction enzymes, namely $A c c \mathrm{I}, B g l \mathrm{II}, B a m \mathrm{HI}, E c o \mathrm{RI}, E c o \mathrm{RV}$, HindIII, XbaI and XhoI.

Genomic DNA digested with each restriction enzyme was electrophoresed through $1 \%$ agarose gel. The gel was soaked in $0.5 \mathrm{M} \mathrm{NaOH}, 1.5 \mathrm{M} \mathrm{NaCl}$ for $30 \mathrm{~min}$ and blotted onto a Hybond- $\mathrm{N}^{+}$nylon membrane (Amersham, UK). The filters were probed with $\alpha-\mathrm{P}^{32} \mathrm{dCTP}$-labeled 
safflower FAD2 DNA fragment. The hybridization was performed in 6x SSPE, 10\% Denhardt's solution, $0.5 \%$ SDS, $100 \mathrm{ug} / \mathrm{mL}$ denatured salmon sperm DNA overnight at $65^{\circ} \mathrm{C}$. After a brief wash in $2 \mathrm{x} \mathrm{SSC} / 0.1 \% \mathrm{SDS}$ at $50^{\circ} \mathrm{C}$, the filter was washed three times in $0.2 \times \mathrm{SSC} / 0.1 \% \mathrm{SDS}$ at $50^{\circ} \mathrm{C}$ for $20 \mathrm{~min}$ each prior to autoradiography.

\section{The isolation of $5^{\prime}$ UTR intron of CtFAD2 genes}

In order to isolate the DNA sequences of an intron that is situated at the $5^{\prime}$ UTR of CtFAD2 genes, the typical splice site (AG:GT) was predicted in the $5^{\prime}$ UTR of each CtFAD2 CDNA sequence, and PCR primers were designed based on the flanking sequences of predicted splice site. The primers are listed in Additional file 1: Table S3. Genomic DNA isolated from safflower genotype SU was used as the template. The amplification was accomplished in $50 \mu \mathrm{L}$ reactions with $100 \mathrm{ng}$ of genomic DNA, $20 \mathrm{pmol}$ of each primer, and a Hotstar (Qiagen, Hilden, Germany) supplied by the manufacturer. PCR temperature cycling was performed as follows: $94^{\circ} \mathrm{C}$ for $15 \mathrm{~min}$ for one cycle, $94^{\circ} \mathrm{C}$ for $30 \mathrm{~s}, 55^{\circ} \mathrm{C}$ for $1 \mathrm{~min}, 72^{\circ} \mathrm{C}$ for $2 \mathrm{~min}$ for 35 cycles; $72^{\circ} \mathrm{C}$ for 10 min using the Kyratec supercycler SC200 (Kyratec, Queensland, Australia). The PCR products were cloned into pGEM-T Easy ${ }^{\circledR}$ (Promega, Madison, WI, USA) following manufacturer's instructions, prior to DNA sequence determination.

\section{Real-Time quantitative PCR}

Gene expression analysis was performed by RT-qPCR using BIORAD CFX96 ${ }^{\mathrm{TM}}$ Real-time PCR detection system and iQTM SYBR ${ }^{\circledR}$ Green Supermix (BioRad, Hercules, CA, USA). Primers with Tm (melting temperature) of about $65^{\circ} \mathrm{C}$ and $19-23 \mathrm{bp}$ in length were designed for genespecific amplification of a product about 100-200 bp fragments (Additional file 1: Table S4). PCR reactions were carried out in 96-well plates. All reactions were performed in triplicates. Reaction mix (10 uL per well) contained $1 \mathrm{x}$ iQTM SYBR ${ }^{\circledR}$ Green Supermix (BioRad, Hercules, CA, USA), $5 \mu \mathrm{M}$ forward and reverse primers, and $400 \mathrm{ng}$ of cDNA. The thermal cycling conditions were $95^{\circ} \mathrm{C}$ for 3 min, followed by 40 cycles of $95^{\circ} \mathrm{C}$ for $10 \mathrm{~s}, 60^{\circ} \mathrm{C}$ for $30 \mathrm{~s}$ and $68^{\circ} \mathrm{C} 30 \mathrm{~s}$. The specificity of the PCR amplification was monitored by melting curve analysis following the final step of the PCR from $60^{\circ} \mathrm{C}$ through $95^{\circ} \mathrm{C}$ at $0.1^{\circ} \mathrm{C} / \mathrm{sec}$. Additionally, PCR products were also checked for purity by agarose gel electrophoresis and confirmed by sequencing. A constitutively expressed $\beta$-ketoacyl-acyl-carrier protein (ACP) synthase II (KASII) gene was used as an endogenous reference. KASII is responsible for the elongation of $\mathrm{C} 16: 0-\mathrm{ACP}$ to $\mathrm{C} 18: 0-\mathrm{ACP}$ in the de novo fatty acid biosynthesis in plants. The suitability of safflower $K A S I I$ gene as an internal reference gene was validated by its high expression stability in various tissues and developmental stages (unpublished data). The data were calibrated relative to the corresponding gene expression level following the $2^{-\Delta \Delta \mathrm{Ct}}$ method for relative quantification [72]. The data were presented as means \pm SD of three reactions performed on independent 96-well plates.

\section{Expression of CtFAD2 genes in Saccharomyces cerevisiae}

The DNA fragment containing the entire open reading frames of safflower CtFAD2 cDNAs were excised from pGEM-Teasy vector as an EcoRI fragment and re-ligated to the corresponding site of the vector pENTR11 (Invitrogen, Carlsbad, CA, USA) and then cloned to the destination expression vector pYES2-DEST52 using the Gateway ${ }^{\circledR}$ Cloning recombination technology (Stratagen, La Jolla, CA, USA) that has GAL1 promoter for inducible gene expression. The gene sequences in these plasmids were each verified by DNA sequence determination. The resulting plasmids and the pYES2-DEST52 vector lacking cDNA insert were introduced into bakers' yeast (Saccharomyces cerevisiae) YPH499 cells by lithium acetate-mediated transformation. Expression of these CtFAD2 genes in yeast cells with or without exogenous fatty acid substrate feeding was essentially as previously described [73]. Each experiment was carried out in triplicate.

\section{Transient expression of CtFAD2 in Nicotiana benthamiana leaves}

Each of the CtFAD2 ORFs was cloned in sense orientation into a modified pORE04 binary vector between the double CaMV-35S promoter and an Agrobacterium tumefaciens NOS terminator containing polyadenylation signal sequence [74]. A vector constitutively expressing the viral suppressor protein, P19, was obtained from Dr Peter Waterhouse's lab. Previous research indicated that the expression of transgenes could be significantly enhanced by the co-expression of P19 to prevent host transgene silencing in $N$. benthamiana leaf-based transient assay [16,75,76]. $A$. tumefaciens strain AGL1 harbouring the 35S:CtFAD2 was grown at $28^{\circ} \mathrm{C}$ with shaking in LB broth supplemented with the $50 \mathrm{mg} / \mathrm{L}$ kanamycin for two days prior to pelleting by centrifugation and resuspension in $1 \mathrm{~mL}$ of infiltration buffer containing $5 \mathrm{mM}$ MES, $5 \mathrm{mM} \mathrm{MgSO}_{4}$ and $100 \mu \mathrm{M}$ acetosyringone and cultured at $28^{\circ} \mathrm{C}$ with shaking for an additional three hours. The $10 \mathrm{x}$ dilution of each culture was then diluted by 10 times with the infiltration buffer and mixed with equal volume of the 35S:P19 culture and infiltrated into the underside of the fully expanded $N$. benthamiana leaves as described by Voinnet et al. [16]. Following a period of 5 days further growth at $24^{\circ} \mathrm{C}$, the infiltrated leaf regions were excised and immediately subjected to fatty acid analysis.

\section{Fatty acid analysis}

Fatty acid methyl esters (FAME) were prepared by transesterification of the total fatty acids in yeast cells, 
obtained as cell pellets after centrifugation of cultures [73], or in N. benthamiana leaf, by adding $750 \mu \mathrm{L}$ of $1 \mathrm{~N}$ $\mathrm{MeOH}-\mathrm{HCl}$ (Supelco) at $80^{\circ} \mathrm{C}$ for minimal $2 \mathrm{hrs}$, then added $500 \mu \mathrm{L}$ of $0.9 \% \mathrm{NaCl}$. FAMEs were extracted with $300 \mu \mathrm{L}$ of hexane, and analysed by GC with Agilent 7890A GC on a 30-m BPX70 column essentially as described before [77] except that the ramping program changed to initial temperature at $150^{\circ} \mathrm{C}$, holding $1 \mathrm{~min}$, ramping $3^{\circ} \mathrm{C} / \mathrm{min}$ to $210^{\circ} \mathrm{C}$, the $50^{\circ} \mathrm{C} / \mathrm{min}$ to $240^{\circ} \mathrm{C}$ for a final holding $2 \mathrm{~min}$. Confirming double bond positions in the FAME by 2,4-dimethyloxazoline (DMOX) modification and GC-MS analysis were carried out same as previously described [77], except with a Shimadzu GC-MS QP2010 Plus on a 30-m BPX70 column. The column temperature was programmed as an initial temperature at $150^{\circ} \mathrm{C}$ for $1 \mathrm{~min}$, ramping at $5^{\circ} \mathrm{C} / \mathrm{min}$ to $200^{\circ} \mathrm{C}$ then $10^{\circ} \mathrm{C} / \mathrm{min}$ to $240^{\circ} \mathrm{C}$ with holding for $5 \mathrm{~min}$. Mass spectra were acquired and processed with GCMS solution software (Shimadzu, Version 2.61). The free fatty acids and FAME standards were purchased from Sigma-Aldrich (St. Louis, MO, USA).

\section{Additional file}

Additional file 1: Figure $\mathbf{S 1}$. Sequence alignment of the putative polypeptides derived from the 11 safflower CtFAD2 and orthologous plant FAD2s. atDES, AAM61113.1; ICDES, ACR15954.1; pfOH:DES, AAC32755.1; plOH, ABQ01458.1; COCONJ, AAK26632.1; haACET, ABC59684.1; rhACET, AAO38035.1; dsACET, AAO38036.1; CaACET ABC00769.1; cPEPOX, CAA76156.1; sIEPOX, AAR23815.1; dCACET, AAO38033.1; dCDES:OH, AAK30206.1; fVACET,AAO38034.1; hhACET, AAO38031.1; boDES, AAC31698.1; haDES-2, AAL68982.1; haDES-3, AAL68983.1; haDES-1, AAL68981.1; ntDES,AAT72296.2; oeDES, AAW63040; siDES, AAF80560.1; ghDES-1, CAA65744.1; ptDES, XP_002297660.1; rCOH, AAC49010.1; cPDES, AAS19533.1; ghDES-4, AAQ16653.1; ghDES-2, CAA71199.1; jCDES, ADB93805.1; luDES,ACF49507.1. Figure S2. Mass spectral identification of DMOX derivatives of $C 18: 2^{\Delta 9(Z), 12(E)}$ and $C 18: 2^{\Delta 9}$ (Z),12(Z) from S. cerevisae expressing safflower CtFAD2-11. Figure S3. Mass spectral identification of DMOX derivatives of crepenynic acid (9-octadecen-12-ynoic acid) from $N$. benthamiana leaves transiently expressing safflower CtFAD2-11 (A). methyl crepenynate standard. Table S1. Oligonucleotide primers used in the 3/RACE of multiple CtFAD2 genes in safflower Table S2. Oligonucleotide primers used for amplification of the entire coding region of CTFAD2 genes in safflower Table S3. Oligonucleotide primers used for the amplification of 5/UTR intron of CtFAD2 genes in safflower. Table S4. Oligonucleotide primers used for RT-qPCR in the expression profile study of safflower CtFAD2 genes.

\section{Abbreviations}

FAD2: Microsomal $\Delta 12$ fatty acid desaturase; CtFAD2: Carthamus tinctorius FAD2; ER: Endoplasmic reticulum; EST: Expressed sequence tag; NCBI: National Center for Biotechnology Information; PCR: Polymerase chain reaction; RT-qPCR: Real time quantitative reverse transcription polymerase chain reaction.

\section{Competing interests}

The authors declared that they have no competing interests.

\section{Authors' contributions}

SC participated in the experimental design, performed experiments, analysed data, and wrote the manuscript. XRZ participated in the experimental design, performed experiments, CCW, AGG and SPS also participated in the experimental design and writing of manuscript, LL participated in manuscript preparation and supervision of experiments, QL participated in the experimental design, performed experiments, supervised all procedures, analysed data and wrote the manuscript. All authors read and approved the final manuscript.

\section{Acknowledgements}

This research project was supported by Australian Grains Research and Development Corporation (GRDC) for financial assistance. Shijiang Cao wishes to thank China Scholarship Council (CSC) for providing scholarship for studying in Australia. We also wish to thank Anu Mathew, Luch Hac, Amratha Ashwin for excellent technical support.

\section{Author details}

'Commonwealth Scientific and Industrial Research Organization Plant Industry, PO Box 1600, Canberra ACT 2601, Australia. ${ }^{2}$ School of Life Sciences, Northeast Normal University, Changchun, China.

Received: 22 August 2012 Accepted: 21 December 2012 Published: 7 January 2013

\section{References}

1. Knowles PF: Safflower. In Oil crops of the world: their breeding and utilization. Edited by Robbelen G, Downey RK, Ashri A. New York: McGraw-Hill; 1989:363-374

2. Vos E: Linoleic acid, "vitamin F6" - is the western world getting too much? probably. Lipid Technology 2003, 15:81-84.

3. Mozaffarian D, Katan MB, Ascherio A, Stampfer MJ, Willett WC: Trans fatty acids and cardiovascular disease. Obstet Gynecol Surv 2006, 61:525-526.

4. Ascherio A, Katan MB, Zock PL, Stampfer MJ, Willett WC: Trans fatty acids and coronary heart disease. New Engl J Med 1999, 340:1994-1998.

5. Karlheinz H: Fats and oils as oleochemical raw materials. Pure App/ Chem 2000, 72:1255-1264.

6. Kinney AJ, Cahoon EB, Hitz WD: Manipulating desaturase activities in transgenic crop plants. Biochem Soc T 2002, 30:1099-1103.

7. Crandall L: Bioplastics: A burgeoning industry. In International News on Fats, Oils and Related Materials. 13th edition.; 2002:626.

8. Browse J, Somerville C: Glycerolipid synthesis: biochemistry and regulation. Annu Rev Plant Physiol Plant Mol Biol 1991, 42:467-506.

9. Wallis JG, Browse J: Mutants of arabidopsis reveal many roles for membrane lipids. Prog Lipid Res 2002, 41:254-278.

10. Miquel M, Browse J: Arabidopsis mutants deficient in polyunsaturated fatty acid synthesis. Biochemical and genetic characterization of a plant oleoyl-phosphatidylcholine desaturase. J Biol Chem 1992, 267:1502-1509.

11. Okuley J, Lightner J, Feldmann K, Yadav N, Lark E, Browse J: Arabidopsis FAD2 gene encodes the enzyme that is essential for polyunsaturated lipid synthesis. Plant Cell 1994, 6:147-158.

12. Shanklin J, Cahoon EB: Desaturation and related modifications of fatty acids. Annu Rev Plant Physiol Plant Mol Biol 1998, 49:611-641.

13. Broun P, Somerville C: Accumulation of ricinoleic, lesquerolic, and densipolic acids in seeds of transgenic arabidopsis plants that express a fatty acyl hydroxylase cDNA from castor bean. Plant Physiol 1997, 113:933-942.

14. Van Der Loo FJ, Broun P, Turner S, Somerville C: An oleate 12-hydroxylase from ricinus communis $L$. Is a fatty acyl desaturase homolog. Proc Natl Acad Sci USA 1995, 92:6743-6747.

15. Lee M, Lenman M, Banaś A, Bafor M, Singh SP, Schweizer M, Nilsson R, Liljenberg C, Dahlqvist A, Gummeson PO, et al: Identification of non-heme diiron proteins that catalyze triple bond and epoxy group formation. Science 1998, 280:915-918.

16. Voinnet $O$, Rivas $S$, Mestre P, Baulcombe D: An enhanced transient expression system in plants based on suppression of gene silencing by the p19 protein of tomato bushy stunt virus. Plant J 2003, 33:949-956.

17. Cahoon EB, Carlson TJ, Ripp KG, Schweiger BJ, Cook GA, Hall SE, Kinney AJ: Biosynthetic origin of conjugated double bonds: production of fatty acid components of high-value drying oils in transgenic soybean embryos. Proc Natl Acad Sci USA 1999, 96:12935-12940.

18. Liu Q, Brubaker CL, Green AG, Marshall DR, Sharp PJ, Singh SP: Evolution of the FAD2-1 fatty acid desaturase $5^{\prime}$ UTR intron and the molecular systematics of Gossypium (Malvaceae). Am J Bot 2001, 88:92-102. 
19. Dyer JM, Chapital DC, Kuan J-CW, Mullen RT, Turner C, McKeon TA, Pepperman AB: Molecular analysis of a bifunctional fatty acid conjugase/ desaturase from tung. Implications for the evolution of plant fatty acid diversity. Plant Physiol 2002, 130:2027-2038.

20. Cahoon EB, Kinney AJ: Dimorphecolic acid is synthesized by the coordinate activities of two divergent delta12-oleic acid desaturases. J Biol Chem 2004, 279:12495-12502.

21. Broun P, Boddupalli S, Somerville C: A bifunctional oleate 12-hydroxylase: desaturase from Lesquerella fendleri. Plant J 1998, 13:201-210.

22. Carlsson AS, Thomaeus S, Hamberg M, Stymne S: Properties of two multifunctional plant fatty acid acetylenase/desaturase enzymes. Eur J Biochem 2004, 271:2991-2997.

23. Mikami K, Murata N: Membrane fluidity and the perception of environmental signals in cyanobacteria and plants. Prog Lipid Res 2003, 42:527-543.

24. Zhang JT, Liu H, Sun J, Li B, Zhu Q, Chen SL, Zhang HX: Arabidopsis fatty acid desaturase FAD2 is required for salt tolerance during seed germination and early seedling growth. PLOS One 2012, 7:e30355.

25. Kirsch C, Hahlbrock K, Somssich IE: Rapid and transient induction of a parsley microsomal delta 12 fatty acid desaturase mRNA by fungal elicitor. Plant Physiol 1997, 115:283-289.

26. Gao QM, Venugopal S, Navarre D, Kachroo A: Low oleic acid-derived repression of jasmonic acid-inducible defense responses requires the WRKY50 and WRKY51 proteins. Plant Physiol 2011, 155:464-476.

27. Kachroo P, Venugopal SC, Navarre DA, Lapchyk L, Kachroo A: Role of salicylic acid and fatty acid desaturation pathways in ssi2-mediated signaling. Plant Physiol 2005, 139:1717-1735.

28. McConn M, Browse J: The critical requirement for linolenic acid is pollen development, not photosynthesis, in an arabidopsis mutant. Plant Cell 1996, 8:403-416.

29. Heppard EP, Kinney AJ, Stecca KL, Miao GH: Developmental and growth temperature regulation of two different microsomal [omega]-6 desaturase genes in soybeans. Plant Physio/ 1996, 110:311-319.

30. Scheffler JA, Sharpe AG, Schmidt H, Sperling P, Parkin IA, Lühs W, Lydiate DJ, Heinz E: Desaturase multigene families of brassica napus arose through genome duplication. Theor App/ Genet 1997, 94:583-591.

31. Liu Q, Singh SP, Brubaker CL, Sharp PJ, Green AG, Marshall DR: Molecular cloning and expression of a cDNA encoding a microsomal $\omega-6$ fatty acid desaturase from cotton (Gossypium hirsutum). Funct Plant Biol 1999, 26:101-106.

32. Pirtle IL, Kongcharoensuntorn W, Nampaisansuk M, Knesek JE, Chapman KD, Pirtle RM: Molecular cloning and functional expression of the gene for a cotton delta-12 fatty acid desaturase (FAD2). Biochim Biophys Acta 2001, 1522:122-129.

33. Zhang DY, Pirtle IL, Park SJ, Nampaisansuk M, Neogi P, Wanjie SW, Pirtle RM, Chapman KD: Identification and expression of a new delta-12 fatty acid desaturase (FAD2-4) gene in upland cotton and its functional expression in yeast and Arabidopsis thaliana plants. Plant Physiol Biochem 2009, 47:462-471.

34. Liu Q, Singh SP, Brubaker CL, Green AG: Cloning and sequence analysis of a novel member (accession No. Y10112) Of the microsomal $\omega-6$ fatty acid desaturase family from cotton (Gossypium hirsutum). Plant Physiol 1999, 120:339.

35. Jung S, Swift D, Sengoku E, Patel M, Teulé F, Powell G, Moore K, Abbott A: The high oleate trait in the cultivated peanut [Arachis hypogaea L.]. I. Isolation and characterization of two genes encoding microsomal oleol-PC desaturases. Mol Gen Genet 2000, 263:796-805.

36. Lopez Y, Nadaf HL, Smith OD, Connell JP, Reddy AS, Fritzf AK: Isolation and characterization of the $\Delta 12$-fatty acid desaturase in peanut (Arachis hypogaea L.) and search for polymorphisms for the high oleate trait in spanish market-type lines. Theor App/ Genet 2000, 101:1131-1138.

37. Krasowska A, Dziadkowiec D, Polinceusz A, Plonka A, Lukaszewicz M: Cloning of flax oleic fatty acid desaturase and its expression in yeast. J Am Oil Chem Soc 2007, 84:809-816.

38. Li LY, Wang XL, Gai JY, Yu DY: Molecular cloning and characterization of a novel microsomal oleate desaturase gene from soybean. J Plant Physiol 2007, 164:1516-1526.

39. Cahoon EB, Schnurr JA, Huffman EA, Minto RE: Fungal responsive fatty acid acetylenases occur widely in evolutionarily distant plant families. Plant J 2003, 34:671-683.

40. McCartney AW, Dyer JM, Dhanoa PK, Kim PK, Andrews DW, McNew JA, Mullen RT: Membrane-bound fatty acid desaturases are inserted co-translationally into the ER and contain different ER retrieval motifs at their carboxy termini. Plant J 2004, 37:156-173.

41. Broun P, Shanklin J, Whittle E, Somerville C: Catalytic plasticity of fatty acid modification enzymes underlying chemical diversity of plant lipids. Science 1998, 282:1315-1317.

42. Dauk M, Lam P, Kunst L, Smith MA: A FAD2 homologue from lesquerella lindheimeri has predominantly fatty acid hydroxylase activity. Plant Sci 2007, 173:43-49.

43. Blacklock BJ, Scheffler BE, Shepard MR, Jayasuriya N, Minto RE: Functional diversity in fungal fatty acid synthesis: the first acetylenase from the pacific golden chanterelle. Cantharellus formosus. J Biol Chem 2010, 285:28442-28449.

44. Tang GQ, Novitzky WP, Griffin CH, Huber SC, Dewey RE: Oleate desaturase enzymes of soybean: evidence of regulation through differential stability and phosphorylation. Plant J 2005, 44:433-446.

45. Qiu X, Reed DW, Hong H, MacKenzie SL, Covello PS: Identification and analysis of a gene from Calendula officinalis encoding a fatty acid conjugase. Plant Physiol 2001, 125:847-855.

46. Kim MJ, Kim H, Shin JS, Chung CH, Ohlrogge JB, Suh MC: Seed-specific expression of sesame microsomal oleic acid desaturase is controlled by combinatorial properties between negative cis-regulatory elements in the SeFAD2 promoter and enhancers in the $5^{\prime}$-UTR intron. Mol Genet Genomics 2006, 276:351-368.

47. Mroczka A, Roberts PD, Fillatti JJ, Wiggins BE, Ulmasov T, Voelker T: An intron sense suppression construct targeting soybean FAD2-1 requires a double-stranded RNA-producing inverted repeat T-DNA insert. Plant Physiol 2010, 153:882-891.

48. Covello PS, Reed DW: Functional expression of the extraplastidial arabidopsis thaliana oleate desaturase gene (FAD2) in Saccharomyces cerevisiae. Plant Physiol 1996, 111:223-226.

49. Hernández ML, Mancha M, Martínez-Rivas JM: Molecular cloning and characterization of genes encoding two microsomal oleate desaturases (FAD2) from olive. Phytochemistry 2005, 66:1417-1426.

50. Fofana B, Duguid S, Cloutier S: Cloning of fatty acid biosynthetic genes $\beta$-ketoacyl CoA synthase, fatty acid elongase, stearoyl-ACP desaturase, and fatty acid desaturase and analysis of expression in the early developmental stages of flax (Linum usitatissimum L.) seeds. Plant Sci 2004, 166:1487-1496.

51. Khadake RM, Ranjekar PK, Harsulkar AM: Cloning of a novel omega-6 desaturase from flax (Linum usitatissimum L.) and its functional analysis in Saccharomyces cerevisiae. Mol Biotechnol 2009, 42:168-174.

52. Martínez-Rivas JM, Sperling P, Lühs W, Heinz E: Spatial and temporal regulation of three different microsomal oleate desaturase genes (FAD2) from normal-type and high-oleic varieties of sunflower (Helianthus annuus L.). Mol Breeding 2001, 8:159-168.

53. Kang $J$, Snapp AR, Lu CF: Identification of three genes encoding microsomal oleate desaturases (FAD2) from the oilseed crop Camelina sativa. Plant Physiol Biochem 2011, 49(2):223-229.

54. Chapman MA, Burke JM: DNA sequence diversity and the origin of cultivated safflower (Carthamus tinctorius L.; asteraceae). BMC Plant Biol 2007, 7:60.

55. Ohlrogge J, Browse J: Lipid biosynthesis. Plant Cell 1995, 7(7):957-970.

56. Sperling $P$, Lee $M$, Girke $T$, Zähringer $U$, Stymne $S$, Heinz E: A bifunctional $\Delta 6$-fatty acyl acetylenase/desaturase from the moss Ceratodon purpureus: a new member of the cytochrome b5 superfamily. Eur J Biochem 2000, 267:3801-3811.

57. Namai T, Kato T, Yamaguchi Y, Hirukawa T: Anti-rice blast activity and resistance induction of $\mathrm{C}-18$ oxygenated fatty acids. Blosci Biotech Bioch 1993, 57:611-613.

58. Minto RE, Blacklock BJ: Biosynthesis and function of polyacetylenes and allied natural products. Prog Lipid Res 2008, 47(4):233-306.

59. Bu'Lock JD, Smith GN: The origin of naturally-occurring acetylenes. J Chem Soc C 1967, :332-336.

60. Barley GC, Jones EHR, Thaller V: Crepenynate as a precursor of falcarinol in carrot tissue culture. In Chemistry and biology of naturally-occurring acetylenes and related compounds. Edited by Lam J, Breteler $\mathrm{H}$, Arnason T, Hansen L. Amsterdam: Elsevier; 1988:85-91.

61. Minto RE, Blacklock BJ: Biosynthesis and function of polyacetylenes and allied natural products. Prog Lipid Res 2008, 47:233-306.

62. Hernández ML, Padilla MN, Sicardo MD, Mancha M, Martínez-Rivas JM: Effect of different environmental stresses on the expression of oleate 
desaturase genes and fatty acid composition in olive fruit. Phytochemistry 2011, 72:178-187.

63. Weber H: Fatty acid-derived signals in plants. Trends Plant Sci 2002, 7:217-224.

64. Cahoon EB, Ripp KG, Hall SE, Kinney AJ: Formation of conjugated delta8, delta10-double bonds by delta12-oleic-acid desaturase-related enzymes: biosynthetic origin of calendic acid. J Biol Chem 2001, 276:2637-2643.

65. Singh SP, Thomaeus S, Lee M, Stymne S, Green AG: Transgenic expression of a $\Delta 12$-epoxygenase gene in Arabidopsis seeds inhibits accumulation of linoleic acid. Planta 2001, 212:872-879.

66. Zhou XR, Singh SP, Liu Q, Green AG: Combined transgenic expression of $\Delta 12$-desaturase and $\Delta 12$-epoxygenase in high linoleic acid seeds leads to increased accumulation of vernolic acid. Funct Plant Biol 2006, 33:585-592.

67. Chapman KD, Austin-Brown S, Sparace SA, Kinney AJ, Ripp KG, Pirtle IL, Pirtle RM: Transgenic cotton plants with increased seed oleic acid content. J Am Oil Chem Soc 2001, 78:941-947.

68. Nam JW, Kappock TJ: Cloning and transcriptional analysis of Crepis alpina fatty acid desaturases affecting the biosynthesis of crepenynic acid. $J$ Exp Bot 2007, 58:1421-1432.

69. Thomas $C A$, Allen EH: An antifungal polyacetylene compound from phytophthora-infected safflower. Phytopathology 1970, 60:261-263.

70. Allen EH, Thomas CA: Trans-trans-3,11-tridecadiene-5,7,9-triyne-1,2-diol, an antifungal polyacetylene from diseased safflower (Carthamus tinctorius). Phytochemistry 1971, 10:1579-1582.

71. Sperling P, Hammer U, Friedt W, Heinz E: High oleic sunflower: studies on composition and desaturation of acyl groups in different lipids and organs. Z Naturforsch C 1990, 45:166-172.

72. Schmittgen TD, Livak KJ: Analyzing real-time PCR data by the comparative C(T) method. Nat Protoc 2008, 3:1101-1108

73. Zhou XR, Robert S, Singh SP, Green AG: Heterologous production of GLA and SDA by expression of an Echium plantagineum $\triangle 6$-desaturase gene. Plant Sci 2006, 170:665-673.

74. Coutu C, Brandle J, Brown D, Brown K, Miki B, Simmonds J, Hegedus D: PORE: a modular binary vector series suited for both monocot and dicot plant transformation. Transgenic Res 2007, 16(6):771-781.

75. Wood CC, Petrie JR, Shrestha P, Mansour MP, Nichols PD, Green AG, Singh SP: A leaf-based assay using interchangeable design principles to rapidly assemble multistep recombinant pathways. Plant Biotechnology J 2009, 7:914-924.

76. Petrie JR, Shrestha P, Liu Q, Mansour MP, Wood CC, Zhou XR, Nichols PD, Green AG, Singh SP: Rapid expression of transgenes driven by seedspecific constructs in leaf tissue: DHA production. Plant Methods 2010, 6:8.

77. Zhou XR, Green AG, Singh SP: Caenorhabditis elegans $\Delta 12$-desaturase FAT-2 is a bifunctional desaturase able to desaturate a diverse range of fatty acid substrates at the $\Delta 12$ and $\Delta 15$ positions. J Biol Chem 2011, 286(51):43644-43650.

doi:10.1186/1471-2229-13-5

Cite this article as: Cao et al: A large and functionally diverse family of Fad2 genes in safflower (Carthamus tinctorius L.). BMC Plant Biology 2013 13:5.

\section{Submit your next manuscript to BioMed Central and take full advantage of:}

- Convenient online submission

- Thorough peer review

- No space constraints or color figure charges

- Immediate publication on acceptance

- Inclusion in PubMed, CAS, Scopus and Google Scholar

- Research which is freely available for redistribution 